\title{
An algorithm for detecting Trichodesmium surface blooms in the South Western Tropical Pacific
}

\author{
C. Dupouy ${ }^{1}$, D. Benielli-Gary ${ }^{2}$, J. Neveux ${ }^{3}$, Y. Dandonneau ${ }^{4}$, and T. K. Westberry ${ }^{5}$ \\ ${ }^{1}$ LOPB - Laboratoire d'Océanographie Physique et Biogéochimique, UMR 6535, CNRS-INSU-IRD-Université de la \\ Méditerranée, 1M213, Centre de Nouméa, B. P. A5, New Caledonia, France \\ ${ }^{2}$ LAM - Laboratoire d'Astrophysique de Marseille, Pôle de l'Étoile Site de Château-Gombert 38, rue Frédéric Joliot-Curie \\ 13388, Marseille Cedex 13, France \\ ${ }^{3}$ UPMC - CNRS, UMR7621, Observatoire Océanologique de Banyuls, Laboratoire d'Océanographie Microbienne, Avenue \\ Fontaulé, 66651 Banyuls sur Mer, France \\ ${ }^{4}$ Université de Paris VI-UPMC-LOCEAN and 14 rue de la Victoire, 91740 Chamarande, France \\ ${ }^{5}$ Dept. Botany Plant Pathology, Oregon State University Corvallis, OR 97331-2902, USA
}

Received: 29 May 2011 - Published in Biogeosciences Discuss.: 15 June 2011

Revised: 22 November 2011 - Accepted: 22 November 2011 - Published: 13 December 2011

\begin{abstract}
Trichodesmium, a major colonial cyanobacterial nitrogen fixer, forms large blooms in $\mathrm{NO}_{3}$-depleted tropical oceans and enhances $\mathrm{CO}_{2}$ sequestration by the ocean due to its ability to fix dissolved dinitrogen. Thus, its importance in $\mathrm{C}$ and $\mathrm{N}$ cycles requires better estimates of its distribution at basin to global scales. However, existing algorithms to detect them from satellite have not yet been successful in the South Western Tropical Pacific (SP). Here, a novel algorithm (TRICHOdesmium SATellite) based on radiance anomaly spectra (RAS) observed in SeaWiFS imagery, is used to detect Trichodesmium during the austral summertime in the SP $\left(5^{\circ} \mathrm{S}-\right.$ $\left.25^{\circ} \mathrm{S} 160^{\circ} \mathrm{E}-170^{\circ} \mathrm{W}\right)$. Selected pixels are characterized by a restricted range of parameters quantifying RAS spectra (e.g. slope, intercept, curvature). The fraction of valid (noncloudy) pixels identified as Trichodesmium surface blooms in the region is low (between 0.01 and $0.2 \%$ ), but is about 100 times higher than deduced from previous algorithms. At daily scales in the SP, this fraction represents a total ocean surface area varying from 16 to $48 \mathrm{~km}^{2}$ in Winter and from 200 to $1000 \mathrm{~km}^{2}$ in Summer (and at monthly scale, from 500 to $1000 \mathrm{~km}^{2}$ in Winter and from 3100 to $10890 \mathrm{~km}^{2}$ in Summer with a maximum of $26432 \mathrm{~km}^{2}$ in January 1999). The daily distribution of Trichodesmium surface accumulations in the SP detected by TRICHOSAT is presented for the period 1998-2010 which demonstrates that the number of selected pixels peaks in November-February each year, consistent with field observations. This approach was validated
\end{abstract}

Correspondence to: C. Dupouy (cecile.dupouy@ird.fr) with in situ observations of Trichodesmium surface accumulations in the Melanesian archipelago around New Caledonia, Vanuatu and Fiji Islands for the same period.

\section{Introduction}

The balance between oceanic $\mathrm{N}_{2}$ fixation and nitrogen losses (denitrification) in the ocean has been postulated to regulate atmospheric $\mathrm{CO}_{2}$ over geological time via the enhancement of biological sequestration of $\mathrm{CO}_{2}$ (Falkowski, 1997; Gruber and Sarmiento, 1997; Deutsch et al., 2007; Capone and Knapp, 2007). Unicellular (Zehr et al., 2001; Montoya et al., 2004; Church et al., 2008, 2009; Zehr et al., 2011) and filamentous cyanobacteria (Carpenter, 1983; Capone et al., 1997, 2005; LaRoche and Breitbarth, 2005; Bonnet et al., 2009; Moisander et al., 2010) incorporate this form of "new" nitrogen $(\mathrm{N})$ into the marine food web of tropical and subtropical oceans (Berman-Frank et al., 2004; Mahaffey et al., 2005; Mulholland, 2007). $\mathrm{N}_{2}$ fixation is considered to be the major source of new $\mathrm{N}$ in stratified, oligotrophic tropical oceans (Capone et al., 1997; Karl et al., 2002). Future change in sea surface temperature (Breitbarth et al., 2006) or/and $\mathrm{CO}_{2}$ concentration are expected to stimulate photosynthesis (C fixation) and $\mathrm{N}_{2}$ fixation by filamentous cyanobacteria, particularly by Trichodesmium spp. (Barcelos et al., 2007; Hutchins et al., 2007; Kranz et al., 2009; Levitan et al., 2010). This enhancement of Trichodesmium growth could compensate the decreased growth of other phytoplankton owing to a presumed decrease of nitrate supply.

Published by Copernicus Publications on behalf of the European Geosciences Union. 
Trichodesmium spp. can form extensive blooms which have been observed for a long time in the South Western Pacific Ocean (SP), particularly in austral summer (Dandonneau and Gohin, 1984). The presence of three major archipelagos (New Caledonia, Vanuatu and Fiji-Tonga in the $\mathrm{SP}$ region $\left(5^{\circ} \mathrm{S}-25^{\circ} \mathrm{S}, 150^{\circ} \mathrm{E}-170^{\circ} \mathrm{W}\right.$, Fig. 1a) and their potential for oceanic iron enrichment from land may trigger these cyanobacterial blooms (Bowman and Lancaster, 1965; Mantas et al., 2011) as Trichodesmium blooms require large quantities of iron (Rubin et al., 2011). The blooms appear as brown or orange meandering patterns around those archipelagos (Dupouy et al., 1988; Dupouy, 1990; Tenório, 2006; Hashihama et al., 2010), are clearly detected from the International Space Shuttle (December 2001 around Tonga Islands), and were recently highlighted by the NASA Ocean Color Website (Feldman et al., 2010). Blooms are also regularly observed in waters of the Dampier Archipelago, the Arafura Sea (Neveux et al., 2006) and off the Great Barrier Reef (Kuchler and Jupp, 1988; Furnas, 1989; Bell et al., 1999). Trichodesmium was reported in the Western North Pacific (Shiozaki et al., 2009; Kitajima et al., 2009; Konno et al., 2010). Nevertheless, observations in the SP contradict the recently published global map of Trichodesmium analogs based on ecosystem model results that indicate a predominance of higher densities in the North Pacific than in the South Western Pacific Ocean (Monteiro et al., 2010).

Estimating the occurrence of Trichodesmium surface blooms from satellite is a major challenge, but will be required for large-scale estimates of nitrogen fixation (e.g., Westberry et al., 2005; Westberry and Siegel, 2006). Regional algorithms have been successfully applied on the Atlantic continental shelf (Subramaniam et al., 2002), off Canary Islands (Ramos et al., 2005) as well as the Indian coast (Sarangi et al., 2004). Global algorithms have also been recently developed for estimating phytoplankton community structure in the surface oceans. The PHYtoplankton SATellite (PHYSAT) algorithm was successful in identifying Synechococcus-like cyanobacteria with maxima in the tropics (Alvain et al., 2005). The Scanning Imaging Absorption Spectrometer for Atmospheric Cartography (SCHIAMACHY) sensor also detects a cyanobacterial signal within the same latitudinal band (Bracher et al., 2009). However, all algorithms generally fail at identifying seasonality of Trichodesmium blooms in the SP and none retrieve the relatively high abundance of Trichodesmium surface blooms expected and observed in situ during austral summer (November to March). Here we develop an algorithm to detect Trichodesmium surface blooms in the SP which is based on SeaWiFS radiance anomalies (similar to PHYSAT) and apply it to SeaWiFS data from 1997-2010.

\section{Material and methods}

\subsection{Data}

\subsubsection{In situ observations}

Bloom observations (Fig. 2) used here include those collected during the maritime survey of the Economic Exclusive Zone of New Caledonia by the French Navy (aerial and shipboard observations) and those obtained during scientific cruises on the R/V Alis between New Caledonia, Vanuatu, Fiji and Wallis and Futuna Islands $\left(15^{\circ} \mathrm{S}\right.$ to $23^{\circ} \mathrm{S}, 160^{\circ} \mathrm{E}$ to $180^{\circ}$ E; Dupouy et al., 2004a). For most of the observations by the French Navy and the R/V Alis, surface water samples were collected with a bucket and preserved onboard in a $4 \%$ formalin solution. Identification of diazotroph morphological groups was made under a Zeiss microscope at IRD Nouméa (LOCEAN laboratory). In addition, more detailed identification and abundance of filamentous diazotrophs was obtained from inverted microscopy between October 2001 and October 2003 (nine Diapalis cruises as part of the DIAzotrophy in a Pacific ZONe program) (Tenório, 2006). In these instances, surface sampling was done with $8 \mathrm{~L}$ Niskin bottles (sometimes with bucket sampling for comparison). During the Diapalis cruises, chlorophyll-and phycoerythrin measurements were also obtained from spectrofluorometry (Lantoine and Neveux, 1997; Neveux et al., 1999; Neveux et al., 2006). A slightly higher concentration of chlorophyll$a$ (Chl- $a$ ) was observed from bucket samples compared to those from Niskin samples.

\subsubsection{Satellite ocean color data}

For the development of the new algorithm, representative global SeaWiFS Level3 data (R2010) were selected for summer and winter seasons, and included normalized water leaving radiance, $\mathrm{nLw}(\lambda)$, at 6 channels $(412,443,490,510$, 555 , and $670 \mathrm{~nm}$ ) as well as SeaWiFS chlorophyll and the diffuse attenuation coefficient at $490 \mathrm{~nm}$ (K490 product). From these data, a Look Up Table (LUT) relating K490 to remote sensing reflectance was created (see Sect. 2.2). However, to avoid compositing artifacts upon application of the LUT, daily SeaWiFS Level-2 GAC (R2010) between $1998-2010$ and covering the Western Pacific Ocean $\left(160^{\circ} \mathrm{E}-\right.$ $160^{\circ} \mathrm{W} / 25^{\circ} \mathrm{N}-25^{\circ} \mathrm{S}$ area), were used.

\subsection{Methods}

The general approach was to define a spectral radiance anomaly from SeaWiFS $n L w(\lambda)$ that was specifically related to Trichodesmium surface blooms. It aimed at removing the first order variability in ocean color caused by chlorophyll concentration while preserving the variability that may be specifically caused by individual phytoplankton species or other optically active components. This objective is similar to the PHYSAT classification method (Alvain et al., 2005) 


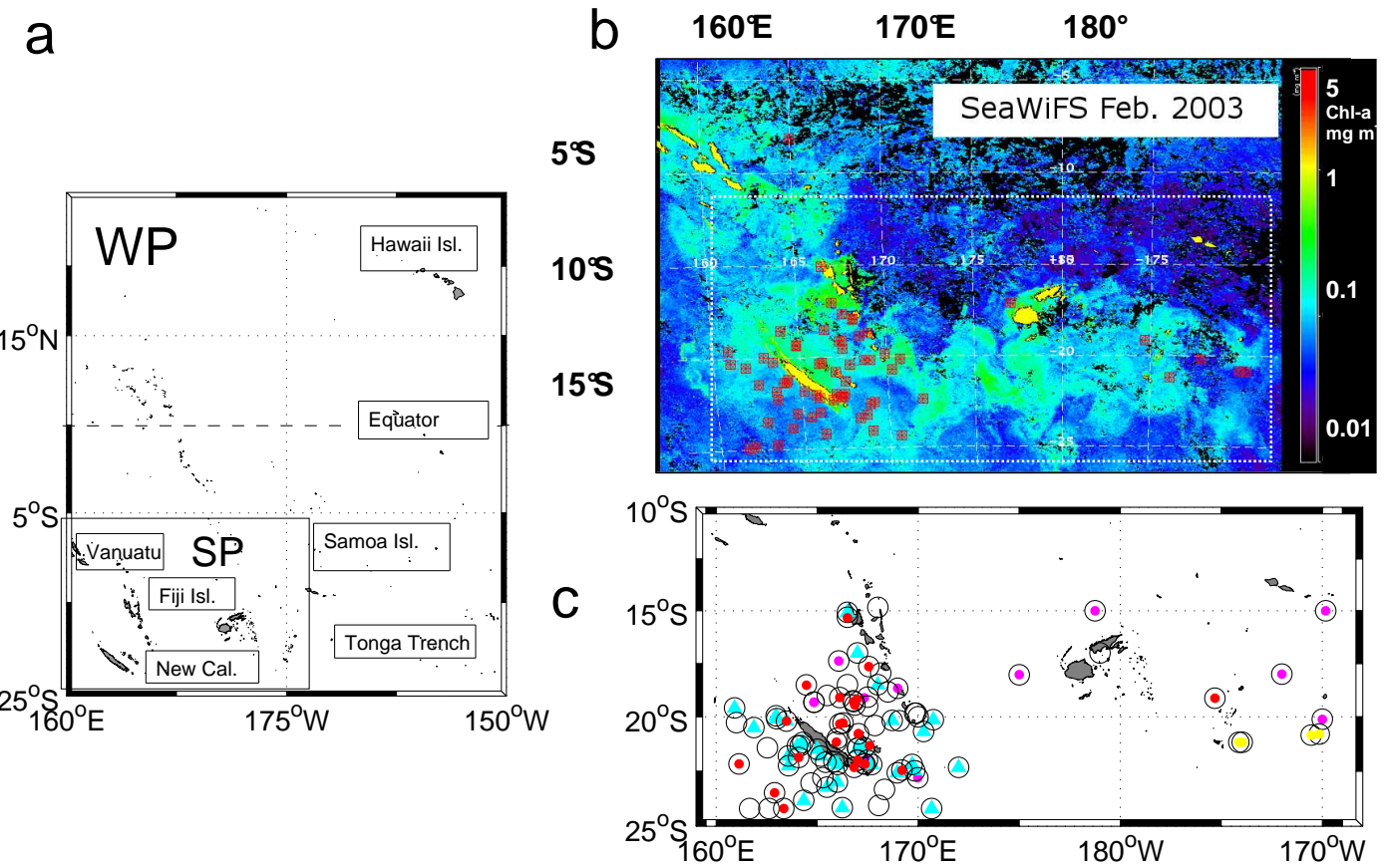

Fig. 1. (a) Map showing limits of the Western Pacific Ocean (WP: $25^{\circ} \mathrm{N}-25^{\circ} \mathrm{S} / 160^{\circ} \mathrm{E}-160^{\circ} \mathrm{W}$ ) and of the Southern Pacific Ocean domains $\left(\mathrm{SP}: 5^{\circ} \mathrm{S}-25^{\circ} \mathrm{S} / 150^{\circ} \mathrm{E}-170^{\circ} \mathrm{W}\right)$. (b) Mean SeaWiFS composite image of chlorophyll- $a\left(\mathrm{mg} \mathrm{m}^{-3}\right)$ in the SP Ocean in February 2003 (austral Summer) with observations of Trichodesmium surface accumulations for December 2002-March 2003. Main islands are indicated in yellow: New Caledonia $\left(20-22^{\circ} \mathrm{S}, 165^{\circ} \mathrm{E}\right)$, Vanuatu $\left(15-20^{\circ} \mathrm{S}, 168^{\circ} \mathrm{E}\right)$, Fiji $\left(17^{\circ} \mathrm{S}, 180^{\circ} \mathrm{E}\right)$ and South Tonga Islands $\left(21^{\circ} \mathrm{S}, 175^{\circ} \mathrm{W}\right)$. (c) French Navy observations of Trichodesmium surface blooms (1998-2010) from Table 1 as empty black circles, with: light blue - aerial observations; red - small form filaments (named T. erythraeum spp. after Tenório, 2006); magenta - mix of small T. erythraeum and long forms (filaments of Trichodesmium thiebautii and T. tenue); yellow - mix of T. tenue and Katagnymene sp.

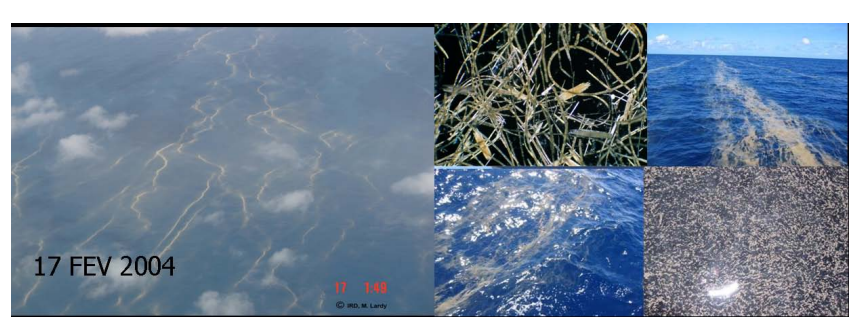

Fig. 2. Photographs of the January-February 2004 Trichodesmium slick as seen from long line airplanes (aerial observation), and from the ship of the French Navy La Glorieuse (shipboard observation with bucket sample) reported in Table 1 .

that was initially developed for discrimination of major phytoplankton groups in Case 1 waters. Waters dominated by diatoms, Prochlorococcus, Synechococcus-like cyanobacteria and haptophytes could thus be classified according to their radiance anomaly spectra (RAS):

$\mathrm{nL}^{*} \mathrm{w}(\lambda)=\mathrm{nLw}(\lambda) /<\mathrm{nLw}(\lambda)>$

where $<\operatorname{nLw}(\lambda)>$ is the expectation of $\mathrm{nLw}$ at a given chlorophyll concentration (Chl- $a$ ), computed as the average of a large global SeaWiFS dataset, and $\mathrm{nL}^{*} \mathrm{w}$ is the radiance anomaly relative to this average. The main advantage of PHYSAT is to provide thresholds allowing characterization of RAS at each pixel. PHYSAT uses a LUT of $<\operatorname{nLw}(\lambda)>$ as a function of Chl- $a$.

Here, we employed a LUT based on the diffuse attenuation coefficient at $490 \mathrm{~nm}$ (SeaWiFS "K490"). K490 has the advantage over chlorophyll of being computed straightforwardly while operational SeaWiFS chlorophyll estimates result from switching between three wavelength ratios. This new LUT was built using four daily, global Level3 images from 15 February, 15 May, 15 August and 15 November 2002, in order to encompass a seasonal cycle. The LUT contains the likelihood of SeaWiFS radiances, noted $<\operatorname{nLw}(\lambda)_{\mathrm{K} 490}>$, for all K490 values in the $0.0186 \mathrm{~m}^{-1}$ to $0.2499 \mathrm{~m}^{-1}$ interval. Radiance anomaly spectra (RAS) are then computed as

$\mathrm{nL}^{*} \mathrm{w}_{\mathrm{K} 490}(\lambda)=\mathrm{nLw}(\lambda) /<\mathrm{nLw}(\lambda)_{\mathrm{K} 490}>$

where $\mathrm{nL}^{*} \mathrm{w}(\lambda)$ are the SeaWiFS radiance estimates. For consistency with past work, we checked that results are equivalent to when Chl- $a$ is used as in PHYSAT. Radiance anomaly spectra $\mathrm{nL}^{*} \mathrm{w}_{K 490}$, hereafter denoted RAS, can thus be considered as equivalent to PHYSAT $n L w^{*}$. 
There is another significant difference between the approach used here compared with the previous PHYSAT effort. Instead of defining RAS thresholds and associating them with pigment classes (and thus, different phytoplankton groups), the new algorithm defines quantitative shape and magnitude criteria of the RAS itself. Eighteen summer scenes around New Caledonia in 2003 and 2004 were selected as they correspond to periods where slicks and high abundance ( $>5000$ trichomes. $\mathrm{L}^{-1}$ ) of Trichodesmium were observed in the surface ocean (Tables 1 and 2). In 2003, SeaWiFS level2-GAC from 6, 13, 18 January, 1, 4, 9, 13, 16, 18, 21, 28 February, 2, 6 March; Yeardays: 6, 13, 18, 32, 35, $40,44,47,49,52,59,62,66)$ corresponded to slick observations by the French Navy from December 2002 to February and March 2003 (Table 1). The gap between December 2002 and February 2003 may be due to the January 2003 cyclone "Beni" that prevented field observations. In 2004, SeaWiFS Level2 GAC from 10, 18, 23 February, and 3, 17 March; Yeardays 41, 48, 53, 63, 77), corresponded to slick observations from January to March 2004 (Table 1). The definition of the RAS of Trichodesmium surface blooms on the Sea-WiFS satellite imagery was generated for pixels around New Caledonia and Fiji from these 18 scenes.

The criteria were chosen in such a way that they permit reconstruction of the RAS spectra. For example, RAS spectra could be described by the coefficients of a polynomial fit (degree 2) (Fig. 3a). We thus retained the following criteria for an objective description of the Trichodesmium RAS (1) slope of the linear fit of RAS vs wavelength (S), and (2) the ordinate of the polynomial fit for which the tangent is parallel to linear fit (Yt). Additional criteria related to the shape of the RAS were defined as the major positive and major negative deviations relative to the second degree polynomial fit (largest "bump" or largest "trough", respectively, Fig. 3a).

\section{Results}

\subsection{Field observations}

Aerial and shipboard visual observations of Trichodesmium surface blooms (Fig. 1b, c) around New Caledonia between 1998 and 2004 are presented in Table 1 and average cruise and transect surface biomass and abundance are presented in Table 2.

Generally, slick sizes were a few tens of meters wide and two to three nautical miles long (shipboard or aircrafts photographs, Fig. 2). Twice in the series (November 1998, November 1999) large areas of $30 \mathrm{~km}^{2}$ were covered by slicks, and once in February 2004, the whole area between New Caledonia and Vanuatu $\left(300 \mathrm{~km}^{2}\right)$ was covered by numerous elongated slicks well detected by longline aircraft. Trichodesmium was responsible for the majority of the slick observations. Only one preserved sample (12 December 2002) contained small pumices originating from Vanuatu or
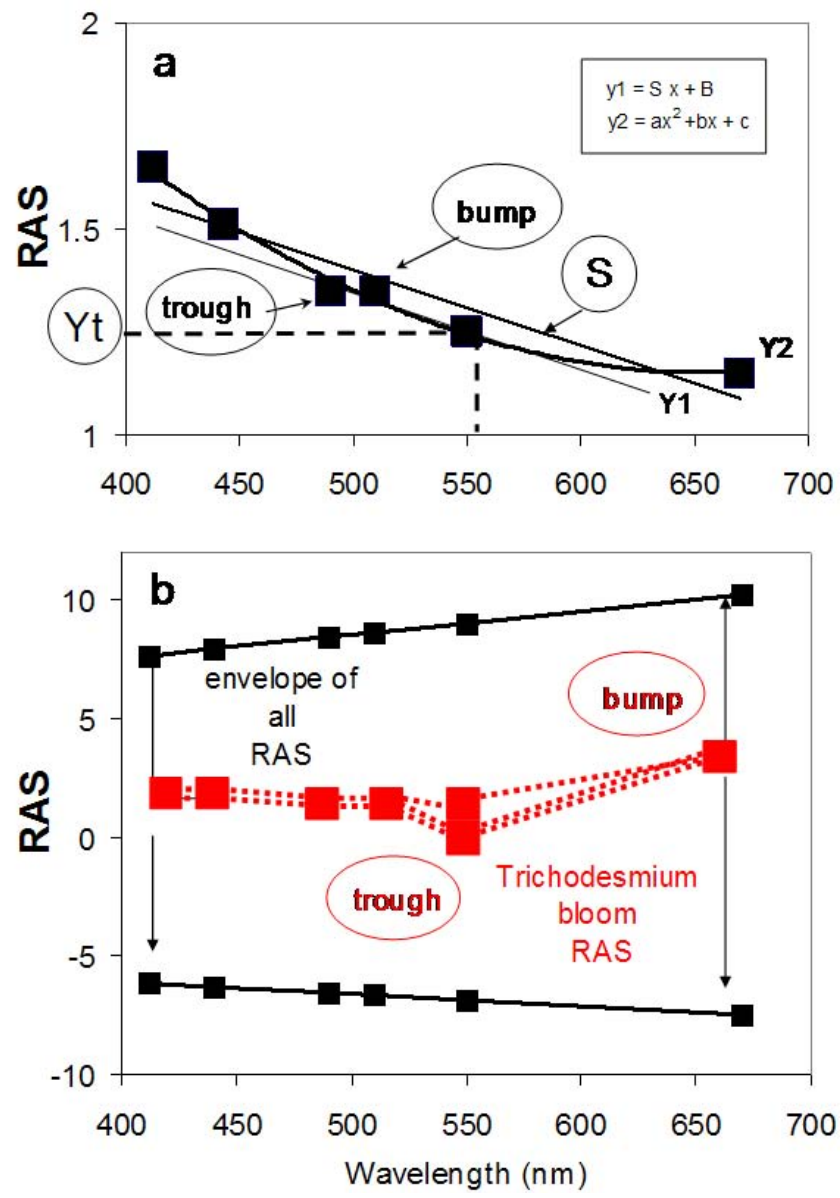

Fig. 3. (a) Descriptive parameters to characterize the radiance anomaly spectra (RAS) relative to K490 for Trichodesmium detection in surface oceanic waters for a SeaWiFS pixel: $\mathrm{S}=$ slope of the linear fit; $\mathrm{Yt}=$ ordinate of the second degree fit for which tangent is parallel to linear fit; "Bump" and "Trough" are the wavelengths of the major positive and major negative deviations relative to the second degree polynomial fit (largest "bump" or largest "trough", respectively) (b) Ranges of S and Yt for all RAS of all SeaWiFS pixels (black). In orange, specific $\mathrm{S}$ and $\mathrm{Yt}$ and positions of bumps and troughs schematically shown for RAS of a Trichodesmium bloom.

Tonga volcanoes which were mixed with the Trichodesmium colonies. Sea-rafted pumice can drift to the west and reach New Caledonia. Some of these rafts reached the Australian coast in October 2002, approximately 7-8 months after a submarine eruption in the Tonga Trench (Bryan et al., 2004). None of the slick observations corresponded to coral spawning which is responsible for fugitive coastal red waters once a year in New Caledonia. The abundance of Trichodesmium spp. in the SP exhibited a strong seasonality as previously pointed out (Dupouy et al., 2004a; Moutin et al., 2005) with the highest number of visual observations $(65 \%$ or 61 over 93 total observations) over the study period (November 1998 to June 2010) occurring between December and February 
Table 1. In situ Trichodesmium bloom observations for the period 1998-2010 in the South Western Tropical Pacific (NC, New Caledonia). Data from aerial observations, and/or bucket sampling from cruises and various transects made by the French Navy ships and the R/V Alis.

\begin{tabular}{|c|c|c|c|c|c|c|c|c|}
\hline Yearday/Year & Date & Latitude & Longitude & $\begin{array}{l}\text { Observed } \\
\text { species }\end{array}$ & $\begin{array}{l}\text { Observed } \\
\text { form }\end{array}$ & $\begin{array}{l}\text { Estimated } \\
\text { Slick size }\end{array}$ & $\begin{array}{l}\text { Location in the } \\
\text { Tropical Pacific }\end{array}$ & $\begin{array}{l}\text { Observation } \\
\text { Mean }\end{array}$ \\
\hline $101 / 1998 * *$ & 11 Apr 1998 & $17^{\circ} \mathrm{S}$ & $179^{\circ} \mathrm{E}$ & T. t. & large col. & und. & Fiji Isl. & $\mathrm{R} / \mathrm{V}$ Revelle, NSF \\
\hline 101/1998* & 11 Apr 1998 & $18^{\circ} \mathrm{S}$ & $172^{\circ} \mathrm{W}$ & T. t. & large col. & und. & Vavau Isl. & F. Navy, J. Cartier \\
\hline $108 / 1998$ & 18 Nov 1998 & $22^{\circ} 35^{\prime} \mathrm{S}$ & $168^{\circ} 58^{\prime} \mathrm{E}$ & und. & und. & nq s. & Walpole Isl. & F. Navy aerial \\
\hline $108 / 1998$ & 18 Nov 1998 & $24^{\circ} 21^{\prime} \mathrm{S}$ & $170^{\circ} 40^{\prime} \mathrm{E}$ & und. & und. & nq s. & SE NC & F. Navy aerial \\
\hline $329 / 1998$ & 25 Nov 1998 & $22^{\circ} 15^{\prime} \mathrm{S}$ & $163^{\circ} 35^{\prime} \mathrm{E}$ & und. & und. & nq s. & W NC & F. Navy aerial \\
\hline $329 / 1998$ & 25 Nov 1998 & $21^{\circ} 20^{\prime} \mathrm{S}$ & $164^{\circ} 10^{\prime} \mathrm{E}$ & und. & und. & nq s. & W NC & F. Navy aerial \\
\hline $329 / 1998$ & 25 Nov 1998 & $19^{\circ} 35^{\prime} \mathrm{S}$ & $160^{\circ} 55^{\prime} \mathrm{E}$ & und. & und. & $30 \mathrm{~km}^{2}$ & NW NC & F. Navy aerial \\
\hline $352 / 1998$ & 18 Dec 1998 & $22^{\circ} 40^{\prime} \mathrm{S}$ & $165^{\circ} 50^{\prime} \mathrm{E}$ & und. & und. & nq s. & SW NC & F. Navy aerial \\
\hline 008/1999 & 8 Jan 1999 & $23^{\circ} \mathrm{S}$ & $166^{\circ} \mathrm{E}$ & und. & und. & nq s. & SW NC & F. Navy aerial \\
\hline 008/1999 & 8 Jan 1999 & $24^{\circ} 10^{\prime} \mathrm{S}$ & $166^{\circ} 15^{\prime} 4 \mathrm{E}$ & und. & und. & nq s. & SW NC & F. Navy aerial \\
\hline 008/1999 & 8 Jan 1999 & $23^{\circ} 50^{\prime} \mathrm{S}$ & $164^{\circ} 20^{\prime} \mathrm{E}$ & und. & und. & nq s. & SW NC & F. Navy aerial \\
\hline 313/1999 & 9 Nov 1999 & $20^{\circ} \mathrm{S}$ & $170^{\circ} \mathrm{E}$ & und. & und. & $30 \mathrm{~km}^{2}$ & Vanuatu to NC & $\mathrm{R} / \mathrm{V}$ Alis (Wespalis) \\
\hline $007 / 2000$ & 7 Jan 2000 & $21^{\circ} 49^{\prime} \mathrm{S}$ & $165^{\circ} 11^{\prime} \mathrm{E}$ & und. & und. & nq s. & W NC & F. Navy aerial \\
\hline $007 / 2000$ & 7 Jan 2000 & $20^{\circ} 30^{\prime} \mathrm{S}$ & $161^{\circ} 52^{\prime} \mathrm{E}$ & und. & und. & nq s. & W NC & F. Navy aerial \\
\hline $363 / 2000$ & $28 \operatorname{Dec} 2000$ & $19^{\circ} 57^{\prime} \mathrm{S}$ & $162^{\circ} 58^{\prime} \mathrm{E}$ & und. & und. & nq s. & Belep & F. Navy aerial \\
\hline $363 / 2000$ & $28 \operatorname{Dec} 2000$ & $21^{\circ} 20^{\prime} \mathrm{S}$ & $164^{\circ} 12^{\prime} \mathrm{E}$ & und. & und. & nq s. & Poum NC & F. Navy aerial \\
\hline $363 / 2000$ & $28 \mathrm{Dec} 2000$ & $21^{\circ} 48^{\prime} \mathrm{S}$ & $163^{\circ} 35^{\prime} \mathrm{E}$ & und. & und. & nq s. & Poum NC & F. Navy aerial \\
\hline 008/2001 & 8 Jan 2001 & $22^{\circ} 20^{\prime} \mathrm{S}$ & $172^{\circ} \mathrm{E}$ & und. & und. & nq s. & Hunter Isl. & F. Navy aerial \\
\hline $018 / 2001$ & 18 Jan 2001 & $21^{\circ} 11^{\prime} \mathrm{S}$ & $164^{\circ} 17^{\prime} \mathrm{E}$ & und. & und. & nq s. & NW Kone, NC & F. Navy aerial \\
\hline $018 / 2001 *$ & 18 Jan 2001 & $18^{\circ} 31^{\prime} \mathrm{S}$ & $164^{\circ} 28^{\prime} \mathrm{E}$ & T. e., T. t., T. Ten. & large col., green & nq s. & Petri Reefs & F. Navy aerial \\
\hline $23 / 2001 *$ & 23 Jan 2001 & $22^{\circ} 11^{\prime} \mathrm{S}$ & $161^{\circ} 79^{\prime} \mathrm{E}$ & T. e. & Isolated fil. & nq s. & Coral Sea & F. Navy aerial \\
\hline $23 / 2001 *$ & 23 Jan 2001 & $22^{\circ} 10^{\prime} \mathrm{S}$ & $161^{\circ} 79^{\prime} \mathrm{E}$ & T. e. & flocks & Shipb. & Coral Sea & F. Navy, La Moqueuse \\
\hline $29 / 2001$ & 29 Jan 2001 & $24^{\circ} 53^{\prime} \mathrm{S}$ & $161^{\circ} 40^{\prime} \mathrm{E}$ & und. & flocks & Shipb. & Coral Sea & F. Navy, La Moqueuse \\
\hline $29 / 2001$ & 29 Jan 2001 & $24^{\circ} 47^{\prime} \mathrm{S}$ & $162^{\circ} 37^{\prime} \mathrm{E}$ & und. & flocks & Shipb. & Coral Sea & F. Navy, La Moqueuse \\
\hline $125 / 2001 *$ & 7 May 2001 & $20^{\circ} 12^{\prime} \mathrm{S}$ & $163^{\circ} 29^{\prime} \mathrm{E}$ & T. e. & green col. & nq s. & Belep & F. Navy aerial \\
\hline $248 / 2001$ & 5 Sep 2001 & $20^{\circ} 08^{\prime} \mathrm{S}$ & $170^{\circ} 45^{\prime} \mathrm{E}$ & und. & und. & nq s. & Anatom & F. Navy aerial \\
\hline $248 / 2001$ & 5 Sep 2001 & $20^{\circ} 41^{\prime} \mathrm{S}$ & $170^{\circ} 16^{\prime} \mathrm{E}$ & und. & und. & nq s. & Anatom & F. Navy aerial \\
\hline $295 / 2001 *$ & 22 Oct 2001 & $22^{\circ} 16^{\prime} \mathrm{S}$ & $167^{\circ} 27^{\prime} \mathrm{E}$ & T. e., T. t., K. & flocks & Shipb. & E NC & R/V Alis (Diapalis 1) \\
\hline $285 / 2001^{*}$ & 3 Nov 2001 & $20^{\circ} 47^{\prime} \mathrm{S}$ & $167^{\circ} 03^{\prime} \mathrm{E}$ & T. e. & grey col. & nq s. & Lifou Isl. & F. Navy aerial \\
\hline $317 / 2001 *$ & 13 Nov 2001 & $22^{\circ} 09^{\prime} \mathrm{S}$ & $167^{\circ} 19^{\prime} \mathrm{E}$ & T. e., T. t., K. & und. & nq s. & East Coast & F. Navy, La Glorieuse \\
\hline $317 / 2001 *$ & 13 Nov 2001 & $22^{\circ} \mathrm{S}$ & $167^{\circ} 29^{\prime} \mathrm{E}$ & T. e., T. t., K. & und. & nq s. & Loyalty Channel & F. Navy, La Moqueuse \\
\hline $030 / 2002 *$ & 30 Jan 2002 & $22^{\circ} 22^{\prime} \mathrm{S}$ & $166^{\circ} 50^{\prime} \mathrm{E}$ & T. e. & green col. & nq $\mathrm{s}$. & Uitoe Pass. & F. Navy aerial \\
\hline $030 / 2002$ & 30 Jan 2002 & $22^{\circ} 12^{\prime} \mathrm{S}$ & $166^{\circ} 01^{\prime} \mathrm{E}$ & und. & und. & nq s. & Uitoe Pass. & F. Navy aerial \\
\hline $039 / 2002$ & 8 Feb 2002 & $21^{\circ} 26^{\prime} \mathrm{S}$ & $162^{\circ} 32^{\prime} \mathrm{E}$ & und. & und. & nq s. & Chesterfields Isl. & $\mathrm{R} / \mathrm{V}$ Alis \\
\hline $111 / 2002 *$ & 21 Apr 2002 & $23^{\circ} 29^{\prime} \mathrm{S}$ & $162^{\circ} 53^{\prime} \mathrm{E}$ & T. e. & orange col. & Shipb. & SW NC & F. Navy, J. Cartier \\
\hline $111 / 2002 *$ & 24 Apr 2002 & $22^{\circ} 28^{\prime} \mathrm{S}$ & $169^{\circ} 12^{\prime} \mathrm{E}$ & T. e. & pink col. & Shipb. & E Walpole Isl. & F. Navy, J. Cartier \\
\hline $189 / 2002 *$ & 8 Jul 2002 & $19^{\circ} 08^{\prime} \mathrm{S}$ & $176^{\circ} 41^{\prime} \mathrm{W}$ & T. e. & grey col. & Shipb. & Fiji Isl. & F. Navy, J. Cartier \\
\hline $197 / 2002$ & $16 \mathrm{Jul} 2002$ & $23^{\circ} 03^{\prime} \mathrm{S}$ & $164^{\circ} 42^{\prime} \mathrm{E}$ & und. & und. & Shipb. & W NC & F. Navy, J. Cartier \\
\hline $344 / 2002 *$ & 10 Dec 2002 & $18^{\circ} 40^{\prime} \mathrm{S}$ & $168^{\circ} 59^{\prime} \mathrm{E}$ & K. + T. e. + T. t. & large col. & nq s. & Erromango Isl. & F. Navy, J. Cartier \\
\hline $345 / 2002$ & 11 Dec 2002 & $20^{\circ} 05^{\prime} \mathrm{S}$ & $167^{\circ} 30^{\prime} \mathrm{E}$ & und. & und. & Shipb. & E Lifou Bay & visual obs \\
\hline $346 / 2002 *$ & $12 \operatorname{Dec} 2002$ & $19^{\circ} 19^{\prime} \mathrm{S}$ & $164^{\circ} 51^{\prime} \mathrm{E}$ & T. e. + K. & green, grey, pummices & Shipb. & Surprises Isl. & F. Navy, La Glorieuse \\
\hline $347 / 2002 *$ & 13 Dec 2002 & $19^{\circ} 07^{\prime} \mathrm{S}$ & $167^{\circ} 20^{\prime} \mathrm{E}$ & K. + T. e. + T. t. & large col. & Shipb. & Erromango Isl. & F. Navy, J. Cartier \\
\hline $350 / 2002 *$ & 16 Dec 2002 & $20^{\circ} 48^{\prime} \mathrm{S}$ & $170^{\circ} 09^{\prime} \mathrm{W}$ & T. ten., K. & grey, long thin fil. & Shipb. & Niue Isl. & $\mathrm{R} / \mathrm{V}$ Alis \\
\hline $350 / 2002 *$ & 16 Dec 2002 & $20^{\circ} 51^{\prime} \mathrm{S}$ & $170^{\circ} 33^{\prime} \mathrm{W}$ & T. ten., $\mathrm{K}$. & grey, long thin fil. & Shipb. & Niue Isl. & $\mathrm{R} / \mathrm{V}$ Alis \\
\hline $352 / 2002 *$ & 18 Dec 2002 & $21^{\circ} 11^{\prime} \mathrm{S}$ & $174^{\circ} 08^{\prime} \mathrm{W}$ & T. ten., K. & grey, long thin fil. & Shipb. & Niue Isl. & $\mathrm{R} / \mathrm{V}$ Alis \\
\hline $352 / 2002 *$ & 18 Dec 2002 & $21^{\circ} 11^{\prime} \mathrm{S}$ & $173^{\circ} 57^{\prime} \mathrm{W}$ & T. ten., $\mathrm{K}$. & grey, long thin fil. & Shipb. & Niue Isl. & $\mathrm{R} / \mathrm{V}$ Alis \\
\hline $359 / 2002$ & $25 \operatorname{Dec} 2002$ & $20^{\circ} 11^{\prime} \mathrm{S}$ & $169^{\circ} 04^{\prime} \mathrm{E}$ & und. & und. & und. & W Tanna Isl. & shipboard \\
\hline $038 / 2003^{*}$ & 5 Feb 2003 & $21^{\circ} \mathrm{S}$ & $166^{\circ} 30^{\prime} \mathrm{E}$ & T.e. & Brown col. & Shipb. & E NC Loyalty Channel & R/V Alis (Diapalis 07) \\
\hline $048 / 2003$ & 17 Feb 2003 & $23^{\circ} 14^{\prime} \mathrm{S}$ & $165^{\circ} 29^{\prime} \mathrm{E}$ & und. & und. & nq s. & Santo Isl. & F. Navy aerial \\
\hline $051 / 2003$ & 20 Feb 2003 & $15^{\circ} 04^{\prime} \mathrm{S}$ & $166^{\circ} 30^{\prime} \mathrm{E}$ & und. & und. & nq s. & W NC & F. Navy aerial \\
\hline $087 / 2003^{*}$ & 28 Mar 2003 & $24^{\circ} 44^{\prime} \mathrm{S}$ & $163^{\circ} 21^{\prime} \mathrm{E}$ & T. e. & pink col. & Shipb. & $\mathrm{S} \mathrm{NC}$ & F. Navy, J. Cartier \\
\hline $115 / 2003$ & 25 Apr 2003 & $17^{\circ} 57^{\prime} \mathrm{S}$ & $168^{\circ} 10^{\prime} \mathrm{E}$ & und. & und. & Shipb. & Efate Isl. & F. Navy, J. Cartier \\
\hline $303 / 2003$ & 30 Oct 2003 & $14^{\circ} 50^{\prime} \mathrm{S}$ & $168^{\circ} \mathrm{E}$ & und. & und. & Shipb. & Bank Isl. & ship of opportunity \\
\hline $308 / 2003^{*}$ & 4 Nov 2003 & $17^{\circ} 39^{\prime} \mathrm{S}$ & $167^{\circ} 33^{\prime} \mathrm{E}$ & T. e. & red col. & Shipb. & Vanuatu & F. Navy, La Moqueuse \\
\hline $309 / 2003$ & 5 Nov 2003 & $20^{\circ} \mathrm{S}$ & $166^{\circ} 30^{\prime} \mathrm{E}$ & und. & und. & Shipb. & Ouvea Isl. & visual obs \\
\hline $343 / 2003$ & 9 Dec 2003 & $20^{\circ} 24^{\prime} \mathrm{S}$ & $167^{\circ} 51^{\prime} \mathrm{E}$ & und. & und. & $300 \mathrm{~km}^{2}$ & $500 \mathrm{~km}$ Lifou-Vanuatu & $\mathrm{R} / \mathrm{V}$ Alis \\
\hline $352 / 2003$ & 18 Dec 2003 & $24^{\circ} 03^{\prime} \mathrm{S}$ & $168^{\circ} 02^{\prime} \mathrm{E}$ & und. & und. & Shipb. & SE NC & F. Navy, La Glorieuse \\
\hline $017 / 2004$ & 17 Jan 2004 & $23^{\circ} 20^{\prime} \mathrm{S}$ & $168^{\circ} 20^{\prime} \mathrm{E}$ & und. & und. & Shipb. & SE NC & F. Navy \\
\hline $023 / 2004$ & 23 Jan 2004 & $20^{\circ} 16^{\prime} \mathrm{S}$ & $161^{\circ} \mathrm{E}$ & und. & und. & Shipb. & NW NC & F. Navy \\
\hline $026 / 2004 *$ & 26 Jan 2004 & $22^{\circ} 11^{\prime} \mathrm{S}$ & $167^{\circ} 22^{\prime} \mathrm{E}$ & T. e. & small orange col. & Shipb. & Loyalty Channel & F. Navy, La Glorieuse \\
\hline $029 / 2004^{*}$ & 29 Jan 2004 & $20^{\circ} 20^{\prime} \mathrm{S}$ & $166^{\circ} 07^{\prime} \mathrm{E}$ & T. e. & Large pink flocks & nq s. & Beautemps-Baupré & R/V Alis (PIL) \\
\hline
\end{tabular}


Table 1. Continued.

\begin{tabular}{|c|c|c|c|c|c|c|c|c|}
\hline Yearday/Year & Date & Latitude & Longitude & $\begin{array}{l}\text { Observed } \\
\text { species }\end{array}$ & $\begin{array}{l}\text { Observed } \\
\text { form }\end{array}$ & $\begin{array}{l}\text { Estimated } \\
\text { Slick size }\end{array}$ & $\begin{array}{l}\text { Location in the } \\
\text { Tropical Pacific }\end{array}$ & $\begin{array}{l}\text { Observation } \\
\text { Mean }\end{array}$ \\
\hline $030 / 2004 *$ & 30 Jan 2004 & $20^{\circ} 17^{\prime} \mathrm{S}$ & $166^{\circ} 17^{\prime} \mathrm{E}$ & T. e. & Large pink flocks & nq s. & $\mathrm{NE} N C$ & $\mathrm{R} / \mathrm{V}$ Alis (PIL) \\
\hline $032 / 2004^{*}$ & 1 Feb 2004 & $21^{\circ} 20^{\prime} \mathrm{S}$ & $167^{\circ} 35^{\prime} \mathrm{E}$ & T. e. & Large pink flocks & nq s. & NE NC, Oua Isl. & $\mathrm{R} / \mathrm{V}$ Alis (PIL) \\
\hline $043 / 2004$ & 12 Feb 2004 & $18^{\circ} 30^{\prime} \mathrm{S}$ & $166^{\circ} 30^{\prime} \mathrm{E}$ & und. & und. & nq s. & W Vanuatu & F. Navy aerial \\
\hline $044 / 2004$ & 13 Feb 2004 & $19^{\circ} 50^{\prime} \mathrm{S}$ & $169^{\circ} 54^{\prime} \mathrm{E}$ & und. & und. & Shipb. & N NC Balabio Isl. & French Navy \\
\hline $047 / 2004$ & 16 Feb 2004 & $18^{\circ} 49^{\prime} \mathrm{S}$ & $168^{\circ} 30^{\prime} \mathrm{E}$ & und. & und. & $\mathrm{km} \mathrm{s}$. & $500 \mathrm{~km} \mathrm{NC-Vanuatu}$ & Air Vanuatu \\
\hline 059/2004* & 28 Feb 2004 & $21^{\circ} 10^{\prime} \mathrm{S}$ & $168^{\circ} 30^{\prime} \mathrm{E}$ & T. e. & orange col. & Shipb. & NE NC coast & $\mathrm{R} / \mathrm{V}$ Alis (Motevas) \\
\hline 059/2004* & $28 \mathrm{Feb} 2004$ & $20^{\circ} 52^{\prime} \mathrm{S}$ & $165^{\circ} 57^{\prime} \mathrm{E}$ & T.e. & brown col. & Shipb. & NE NC coast & $\mathrm{R} / \mathrm{V}$ Alis (Motevas) \\
\hline $060 / 2004^{*}$ & 29 Feb 2004 & $18^{\circ} 33^{\prime} \mathrm{S}$ & $166^{\circ} 03^{\prime} \mathrm{E}$ & T. t. + K. & floating flocks & Shipb. & Vanuatu & $\mathrm{R} / \mathrm{V}$ Alis (Motevas) \\
\hline $060 / 2004^{*}$ & 29 Feb 2004 & $18^{\circ} 20^{\prime} \mathrm{S}$ & $166^{\circ} 02^{\prime} \mathrm{E}$ & T. t. + K. & green tufts long fil. & Shipb. & Vanuatu & $\mathrm{R} / \mathrm{V}$ Alis (Motevas) \\
\hline $060 / 2004 *$ & 29 Feb 2004 & $17^{\circ} 23^{\prime} \mathrm{S}$ & $166^{\circ} 04^{\prime} \mathrm{E}$ & T. t. + K. & large puffs/tufts, flocks & Shipb. & Vanuatu & $\mathrm{R} / \mathrm{V}$ Alis (Motevas) \\
\hline $061 / 2004 *$ & 1 Mar 2004 & $22^{\circ} \mathrm{S}$ & $167^{\circ} \mathrm{E}$ & T. e. & White col. & Shipb. & Yate Barrier Reef & F. Navy \\
\hline $064 / 2004$ & 4 Mar 2004 & $20^{\circ} 09^{\prime} \mathrm{S}$ & $168^{\circ} 43^{\prime} \mathrm{E}$ & und. & und. & nq s. & Vanuatu & F. Navy aerial \\
\hline $089 / 2004$ & 29 Mar 2004 & $17^{\circ} \mathrm{S}$ & $167^{\circ} \mathrm{E}$ & und. & und. & nq s. & Masquilignes Isl. N Vanuatu & Air Vanuatu \\
\hline $117 / 2004$ & 26 Apr 2004 & $20^{\circ} 09^{\prime} \mathrm{S}$ & $163^{\circ} 01^{\prime} \mathrm{E}$ & und. & und. & nq s. & Aircraft & F. Navy aerial \\
\hline $275 / 2004$ & 1 Oct 2004 & $21^{\circ} 30^{\prime} \mathrm{S}$ & $165^{\prime} \mathrm{E}$ & und. & und. & nq s. & Lifou 1sl. & F. Navy aerial \\
\hline $316 / 2004$ & 11 Nov 2004 & $18^{\circ} 30^{\prime} \mathrm{S}$ & $168^{\prime} \mathrm{E}$ & und. & und. & nq s. & Vanuatu & F. Navy aerial \\
\hline $347 / 2004$ & 12 Dec 2004 & $22^{\circ} 29^{\prime} \mathrm{S}$ & $169^{\circ} 50^{\prime} \mathrm{E}$ & und. & und. & nq s. & E Pine Isl. , Walpole Isl. & F. Navy aerial \\
\hline $033 / 2005^{*}$ & 2 Feb 2005 & $20^{\circ} 07^{\prime} \mathrm{S}$ & $170^{\circ} \mathrm{W}$ & T. e. +T. t. + T. Cont. & und. & und. & East Tonga Isl. & Hashihima et al., 2010 \\
\hline $040 / 2005^{*}$ & 9 Feb 2005 & $18^{\circ} 02^{\prime} \mathrm{S}$ & $175^{\circ} \mathrm{E}$ & T. e. +T. t. + T. Cont. & und. & und. & W Fiji Isl. & Hashihima et al., 2010 \\
\hline 090/2007* & 30 Mar 2007 & $22^{\circ} 49^{\prime} \mathrm{S}$ & $169^{\circ} 59^{\prime} \mathrm{E}$ & T. e. + K. + T. C. & Floating puffs & Shipb. & Walpole Isl. & R/V Kilo Moana cruise \\
\hline $089 / 2007 *$ & 8 Apr 2007 & $15^{\circ} \mathrm{S}$ & $169^{\circ} 59^{\prime} \mathrm{W}$ & T. e. + K. + T. C. & Floating puffs & Shipb. & N. Fiji Isl. & R/V Kilo Moana cruise \\
\hline $102 / 2007 *$ & 12 Apr 2007 & $14^{\circ} 59^{\prime} \mathrm{S}$ & $178^{\circ} 45^{\prime} \mathrm{E}$ & T. e. + K. + T. C. & Surface Bloom & Shipb. & North East Fiji & R/V Kilo Moana cruise \\
\hline $062 / 2010 *$ & 3 Mar 2010 & $19^{\circ} 11^{\prime} \mathrm{S}$ & $166^{\circ} 57^{\prime} \mathrm{E}$ & T. e. & orange col. & nq s. & Beautemps- Beaupré Reef & F. Navy aerial \\
\hline $062 / 2010 *$ & 3 Mar 2010 & $19^{\circ} 06^{\prime} \mathrm{S}$ & $166^{\circ} 07^{\prime} \mathrm{E}$ & T. e. & orange col. & nq s. & Beautemps- Beaupré Reef & F. Navy, J. Cartier \\
\hline $102 / 2010$ & 12 Apr 2010 & $22^{\circ} 48^{\prime} \mathrm{S}$ & $165^{\circ} 17^{\prime} \mathrm{S}$ & und. & und. & Shipb. & W NC & Ship obs \\
\hline $102 / 2010$ & 12 Apr 2010 & $22^{\circ} 06^{\prime} \mathrm{S}$ & $165^{\circ} 26^{\prime} \mathrm{E}$ & und. & und. & Shipb. & idem & Ship obs \\
\hline $141 / 2010^{*}$ & 21 May 2010 & $15^{\circ} 19^{\prime} \mathrm{S}$ & $166^{\circ} 30^{\prime} \mathrm{E}$ & T. e. & orange col. & Shipb. & Sabine Bank & $\mathrm{R} / \mathrm{V}$ Alis (Geodeva4) \\
\hline $300 / 2010$ & 27 Oct 2010 & $19^{\circ} \mathrm{S}$ & $165^{\circ} 30^{\prime} \mathrm{E}$ & und. & und. & Shipb. & W NC & Ship obs \\
\hline $301 / 2010$ & 28 Oct 2010 & $22^{\circ} \mathrm{S}$ & $167^{\circ} \mathrm{E}$ & und. & und. & Shipb. & W NC & Ship obs \\
\hline $307 / 2010 *$ & 3 Nov 2010 & $19^{\circ} 15^{\prime} \mathrm{S}$ & $166^{\circ} 47^{\prime} \mathrm{E}$ & T. e. & orange col. & Shipb. & SE Vanuatu & F. Navy, R/V Prairial \\
\hline $307 / 2010^{*}$ & 3 Nov 2010 & $19^{\circ} 27^{\prime} \mathrm{S}$ & $166^{\circ} 52^{\prime} \mathrm{E}$ & T. e. & orange col. & Shipb. & SE Vanuatu & F. Navy, R/V Prairial \\
\hline $339 / 2010$ & 4 Dec 2010 & $22^{\circ} 12^{\prime} \mathrm{S}$ & $169^{\circ} 42^{\prime} \mathrm{E}$ & und. & und. & nq s. & SE NC & F. Navy aerial \\
\hline $339 / 2010$ & 4 Dec 2010 & $21^{\circ} 26^{\prime} \mathrm{S}$ & $167^{\circ} 19^{\prime} \mathrm{E}$ & und. & und. & nq s. & W NC & F. Navy aerial \\
\hline $339 / 2010$ & 4 Dec 2010 & $22^{\circ} 12^{\prime} \mathrm{S}$ & $167^{\circ} 42^{\prime} \mathrm{E}$ & und. & und. & nq s. & W NC & F. Navy aerial \\
\hline
\end{tabular}

Abreviations: T. e., T. t. and T. ten., K., for Trichodesmium erythraeum, thiebautii, and tenue, Katagnymene spp., respectively. Col. = colonies; fil. = filaments. nq s.: nautical miles slicks, shipb.: Shipboard observations. * Ground-truthed (microscopy) aerial or shipboard observations are indicated by an asterisk, ** E. J. Carpenter, personal communication, 1998

(Table 1). Surface blooms were rarely detected in situ during winter (only one observation of slicks was available in July 2002, at the west of Fiji Islands, Table 1). Nearly every year, surface blooms developed between New Caledonia and Vanuatu and one of these blooms was exceptionally well tracked (February 2004, Table 1). Slicks were first observed in November 2003 near Vanuatu $\left(17.65^{\circ} \mathrm{S}\right.$, $\left.167.56^{\circ} \mathrm{E}\right)$, in December 2003 at the north and south of New Caledonia, in January (on 26 at $22^{\circ} \mathrm{S}$ and on 29-30 at $20.33^{\circ} \mathrm{S} / 166.12^{\circ} \mathrm{E}$ ) then in February 2004 (on 1st around New Caledonia and on 12-16 near Vanuatu and again North of New Caledonia at $19.49 .9^{\circ} \mathrm{S}, 169.54^{\circ} \mathrm{E}$ ). The surface bloom persisted after heavy rains and wind-mixing by Category 4 cyclone Ivy on 24 February. Later, it was detected near New Caledonia (on 26 February), and under calm meteorological and sea conditions. Its slick was sampled on 28 February and showed an orange surface scum (around $21^{\circ} \mathrm{S}$, New Caledonia) or large flocks of dead colonies (at $18.55^{\circ} \mathrm{S}, 166.05^{\circ} \mathrm{E}$, Vanuatu) which disappeared in the evening $\left(17.38^{\circ} \mathrm{S}, 166.07^{\circ} \mathrm{E}\right)$ (Motevas cruise, Table 2). In March, white senescent $T$. erythraeum (1-4 March, at $22^{\circ} \mathrm{S}$, $167^{\circ} \mathrm{E}$ and $20.16^{\circ} \mathrm{S} / 168.71^{\circ} \mathrm{E}$ ) and remnant coastal slicks on (29 March) were seen near Vanuatu $\left(17^{\circ} \mathrm{S}, 167^{\circ} \mathrm{E}\right)$. In
April, remnant slicks were seen nearby Lifou island at the east of the "Grande terre" of New Caledonia (Table 1). Surface slicks were also observed in summer during a watercolumn observational program in the Loyalty Channel (12 October 2001, 5 February 2003 and 2004, see Tables 1 and 2).

Among the pelagic species described in the Pacific region (Revelante and Gilmartin, 1982; Carpenter et al., 1993), a high morphological diversity of filamentous cyanobacteria as described by Lundgren et al. (2005) and Tenório (2006) was observed in our surface samples (Fig. 1c). Colonies were composed of small rafts from 10 to 50 filaments, small in length (noted T. erythraeum), or long and twisted rafts (noted T. thiebautii) of 50-100 filaments. Long, curved filaments composed of cells larger than long, and some thinner filaments with cells 5-6x longer than wide were noted $K$. pelagica and $T$. tenue, respectively. The distribution of these four morphotypes is shown in Fig. 1c. There is no evidence that the same Trichodesmium genus or species is widespread throughout the tropics contrary to Katagnymene sp. (Lundgren et al., 2005). We tended to find T. erythraeum near the New Caledonia mainland, but elsewhere most of the morphotypes were mixed. During the peak observed 
Table 2. Average in situ observations in the surface layer ( $3 \mathrm{~m}$ Niskin bottle) of filamentous cyanobacteria during the DIAPAZON Program from Tenório (2006), the Motevas cruise, and in surface bucket samples during the transects made on the French Navy ships from New Caledonia to $\operatorname{Tr}$ 1: Vanuatu, $\operatorname{Tr}$ 2: Walpole, Tr 3: Vanuatu, $\operatorname{Tr}$ 4: Vanuatu, Tr 5: Fiji, Vanuatu, Tr 6: Wallis and Futuna and Vanuatu. Chl$a=$ chlorophyll- $a$; PE $=$ phycoerythrin; Chl $-a>10=$ percentage of Chl- $a$ associated with the $>10 \mu \mathrm{m}$ fraction, $\mathrm{Cp}=$ attenuation coefficient $\left(\mathrm{m}^{-1}\right)$. Und: undetermined.

\begin{tabular}{|c|c|c|c|c|c|c|c|c|}
\hline Yearday/Year & $\begin{array}{l}\text { date } \\
\mathrm{DD} / \mathrm{MM} / \mathrm{YY}\end{array}$ & $\begin{array}{l}\text { cruise } \\
\text { name }\end{array}$ & $\begin{array}{l}\text { Chl- } a \\
\left(\mathrm{mg} \mathrm{m}^{-3}\right)\end{array}$ & $\begin{array}{l}\mathrm{PE} \\
\left(\mathrm{mg} \mathrm{m}^{-3}\right)\end{array}$ & $\begin{array}{l}\text { Chl- } a>10 \\
(\%)\end{array}$ & $\begin{array}{l}\text { Beam attenuation } \\
\left(\mathrm{m}^{-1}\right)\end{array}$ & $\begin{array}{l}\text { Trich. Ab. } \\
\text { (Trichomes.L } L^{-1} \text { ) }\end{array}$ & Bloom obs \\
\hline $105 / 1998$ & 15-April 1998 & Trichonesia & 0.14 & und. & 20 & und. & 298 (10000 Fiji Isl.)* & Shipboard \\
\hline $295-304 / 2001$ & 22-31 October 2001 & Diapalis01 & 0.14 & 0.16 & 16 & und. & 411 & Shipboard \\
\hline $344-356 / 2001$ & 10-22 December 2001 & Diapalis02 & 0.13 & 0.15 & 10 & 0.072 & 474 & no \\
\hline $15-22 / 2002$ & 15-22 January 2002 & Diapalis03 & 0.1 & 0.09 & 8.8 & 0.062 & 401 & no \\
\hline $92-99 / 2002$ & 2-9 April 2002 & Diapalis04 & 0.11 & 0.1 & 9 & 0.059 & 347 & no \\
\hline $133-144 / 2002$ & 13-14 May 2002 & Transect 1 & 0.26 & 0.83 & und. & und. & und. & no \\
\hline 141-148/2002 & 21-28 May 2002 & Diapalis05 & 0.11 & 0.23 & 9.3 & 0.046 & $300(1000 * *)$ & no \\
\hline $217-224 / 2002$ & 5-12 August 2002 & Diapalis06 & 0.22 & 0.19 & 9 & 0.069 & 76 & no \\
\hline $34-43 / 2003$ & $3-12$ February 2003 & Diapalis07 & 0.23 & 0.6 & 53 & 0.1 & $>6000$ & Shipboard \\
\hline $59 / 2003$ & 28 February 2003 & Transect 2 & 0.29 & 0.45 & und. & und. & und. & und. \\
\hline $71-80 / 2003$ & 12-21 March 2003 & Transect 3 & 0.29 & 0.33 & und. & und. & und. & und. \\
\hline $107-115 / 2003$ & 17-25 April 2003 & Transect 4 & 0.21 & 0.53 & und. & und. & und. & no \\
\hline $112-115 / 2003$ & 22-25 April 2003 & Transect 5 & 0.18 & 0.44 & und. & und. & und. & no \\
\hline $161-165 / 2003$ & 9-14 Juni 2003 & Diapalis08 & 0.2 & 0.25 & 7 & 0.051 & 300 & no \\
\hline $188-208 / 2003$ & 7-27 July 2003 & Transect 6 & 0.25 & 0.09 & und. & und. & und. & no \\
\hline $281-289 / 2003$ & 8-16 October 2003 & Diapalis09 & 0.099 & 0.08 & 9 & 0.057 & 9 & no \\
\hline $59-60 / 2004$ & 28-29 February 2004 (Niskin) & Motevas & 0.48 & 0.37 & 70 & und. & 4000 & Shipboard \\
\hline $59-60 / 2004$ & 28-29 February 2004 (Bucket) & Motevas & 0.84 & 3.4 & 70 & und. & 6500 & Shipboard \\
\hline
\end{tabular}

* E. J. Carpenter, personal communication, 1998, ** 1000 in a slick North of Lifou.

Trichodesmium abundance in 2004 along a transect from New Caledonia $\left(20-21^{\circ} \mathrm{S}\right)$ to Vanuatu $\left(19^{\circ} \mathrm{S}-17^{\circ} \mathrm{S}\right)$, short tufts of T. erythraeum were observed at the beginning of the transect (NC), while a mix of T. thiebautii (50\%), T. tenue (25\%), T. erythraeum (15\%), and Katagnymene pelagica and spiralis $(10 \%)$ dominated populations at the end (Vanuatu). This population change was also seen in change in the fluorescence excitation spectra of phycoerythrin as in Neveux et al. (2006). Mixed assemblages of T. erythraeum, T. thiebautii, and T. contortum filaments were also observed on the 2 and 9 February 2005 around Fiji Islands (Hashihama et al., 2010), while floating puffs and tufts of mixed taxonomy were observed in March 2007 (Biegala, pers. com.). Only $K$. pelagica $+T$. tenue colonies were found isolated near Niue Island at $19^{\circ} 2^{\prime} \mathrm{S} 169^{\circ} 52^{\prime} \mathrm{W}$ (Table 1, Fig. 1c).

Surface Trichodesmium abundance and associated biomass (Chl $-a>10 \mu \mathrm{m}$ ) in the Loyalty Channel and along transects between New Caledonia, Vanuatu or Wallis and Futuna indicated a strong seasonal variability (Table 2). Low filament densities characterized the end of the bloom season in April 1998 when Trichodesmium contribution to biomass was reduced (Dupouy et al., 2000; Campbell et al., 2006), as in April 2002 and 2003 (Table 2). High abundance was never observed in May, except in 2002 along transect 1 , where phycoerythrin spectral characteristics confirmed the dominance of Trichodesmium between New Caledonia and Vanuatu. At the beginning of the summer season (October), densities were high in 2001 but not in 2003. Trichodesmium abundance was always higher in summer, except during two 2001-2002 Diapalis cruises (December, January). During
Motevas in February 2004 (Dupouy et al., 2004b, 2008), the light beam attenuation was high $\left(0.1 \mathrm{~m}^{-1}\right)$, and a maximal Trichodesmium contribution to Chl- $a$ of $50-70 \%$ coincident with a high density of 5000 filaments. $L^{-1}$ was observed with maximal biomass of 1.9 and $14 \mathrm{mg} \mathrm{m}^{-3}$ for Chl- $a$ and phycoerythrin, respectively. Densities were similar to those measured during February 2003 in Diapalis 07 (Tenório, 2006; Neveux et al., 2006; Masotti et al., 2007). The determination of biomass in surface slicks is challenging and requires a specific thin-layer surface sampler. Nevertheless, Chl- $a$ concentrations up to $2 \mathrm{mg} \mathrm{m}^{-3}$ in the ocean and $3 \mathrm{~g} \mathrm{~m}^{-3}$ in the New Caledonia lagoon were observed (Tenório, 2006). In winter, Trichodesmium abundance was always low and relatively high chlorophyll values were associated with small size algae (low values of the Chl- $a$ fraction above $10 \mu \mathrm{m}$ ).

\subsection{Detection of Trichodesmium in satellite data}

In summer 2003 and 2004, the majority of pixels for the region around New Caledonia exhibited similar RAS. These RAS characteristics were examined under the assumption that instances where Chl- $a>0.2 \mathrm{mg} \mathrm{m}^{-3}$ were dominated by Trichodesmium while those with $<0.2 \mathrm{mg} \mathrm{m}^{-3}$ were not, similar to in situ observations. For Chl- $a>0.2 \mathrm{mg} \mathrm{m}^{-3}$, linear slopes of the RAS varied between -0.005 and 0.008 , and the tangent ordinate $\mathrm{Yt}$ varied within a range of 0.8 to 1.4. For Chl- $a<0.2 \mathrm{mg} \mathrm{m}^{-3}$, the RAS slope varied from -0.005 to 0.012 , while $\mathrm{Yt}$ also varied between 0.8 to 1.4 . A narrow range of slopes $\mathrm{S}$ (from -0.0019 to -0.0017 ) and intercepts 
a

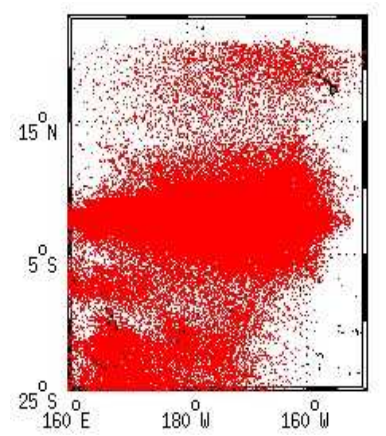

b

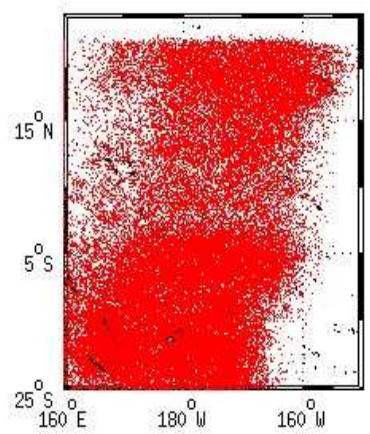

C

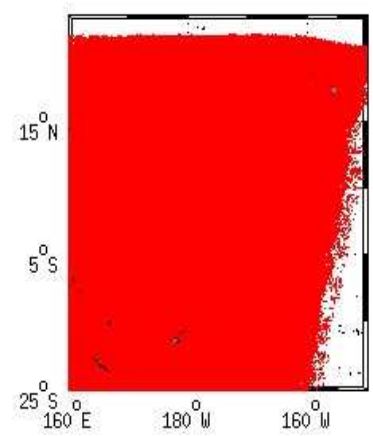

d

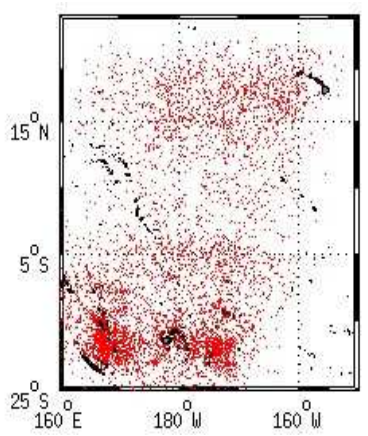

Fig. 4. Application of the TRICHOSAT algorithm to satellite data (1997 to 2010) over the large box corresponding to the Western Pacific Ocean (WP: $25^{\circ} \mathrm{N}-25^{\circ} \mathrm{S} / 160^{\circ} \mathrm{E}-160^{\circ} \mathrm{W}$ ) for the interseason (April-May and October) (a) all pixels for which Chl- $a>0.2 \mathrm{mg} \mathrm{m}^{-3}$; (b) pixels satisfying the slope and intercept criteria of the RAS; (c) pixels satisfying the shape criteria, (d) pixels satisfying all criteria. The main islands of New Caledonia at $20^{\circ} \mathrm{S}$, Vanuatu at $15^{\circ} \mathrm{S}$, Fiji Islands at $17^{\circ} \mathrm{S}$, Hawaii Islands at $20^{\circ} \mathrm{N}$ are indicated in black Fig. $4 \mathrm{~d}$.

Yt (0.9725-1.0175) characterized Trichodesmium pixels (envelope in orange, Fig. 3b) compared to the large range of $\mathrm{S}$ and Yt of RAS of all other pixels (envelope in black, Fig. 1b). These ranges corresponded to low but not minimal values of the total slope range $(-0.005$ to +0.012$)$, and to a narrow interval of values of the total Yt range (0.8 to 1.4) (Fig. 3b). Moreover, it was found that RAS of pixels corresponding to Trichodesmium surface blooms had a specific shape. These RAS spectra were characterized by: (1) lack of a "bump" at $555 \mathrm{~nm}$ (flat RAS or trough at $555 \mathrm{~nm}$ ) and (2) always a "bump" at $670 \mathrm{~nm}$ and never a trough at $670 \mathrm{~nm}$ (Fig. 3b). Criteria for 412, 443, 490 and $510 \mathrm{~nm}$ were rather neutral. No weighting of these criteria was necessary for the selection of pixels which approach this ideal shape. The criteria of the RAS for the $555 \mathrm{~nm}$ and $670 \mathrm{~nm}$ SeaWiFS channels were essential while the criteria for other wavelengths were less useful. On the contrary, quantitative criteria of the RAS defined as in Fig. 3b were discriminant. Ultimately, it appeared that the special RAS shape and quantitative criteria was independent of the Chl- $a$ concentration in the pixels.

The application of Trichodesmium RAS criteria (TRICHOdesmium SATellite algorithm: TRICHOSAT) to all SeaWiFS Level2 data from 1997 to 2010 within the whole Western Pacific ocean region $\left(25^{\circ} \mathrm{N}-25^{\circ} \mathrm{S}\right.$ and $160^{\circ} \mathrm{E}-$ $160^{\circ} \mathrm{W}$, noted WP) is shown as composites in Fig. 4 for the interseason (April-May, October). The application of the shape criteria and the quantitative criteria separately were not sufficient to discriminate Trichodesmium surface bloom pixels (Fig. 4b and c). The TRICHOSAT algorithm finally selected the pixels which satisfy both criteria (Fig. 4d), i.e. the intersection of the two groups selected by the shape criteria and the quantitative criteria. The resulting composite Fig. $4 \mathrm{~d}$ shows that the majority of selected Trichodesmium pixels is in the SP. Note that the algorithm selection differs markedly from the distribution of pixels with Chl- $a$ concentration greater than $0.2 \mathrm{mg} \mathrm{m}^{-3}$ (Fig. 4a) as there are no Trichodesmium pixels in the extensive equatorial upwelling maximum, and only few pixels in the northern part of the Pacific Ocean. Trichodesmium flagged pixels are not necessarily associated with high chlorophyll content pixels, similar to Westberry and Siegel (2006).

In order to get statistically coherent results for the comparison of the percentage of Trichodesmium pixels identified for each year and season, it was verified that a sufficient number of processed SeaWiFS Level2 GAC images $\left(5 \times 10^{4}-\right.$ $1 \times 10^{6}$ pixels with quasi equivalent numbers of non-cloudy valid pixels for each season) were analyzed (Fig. 5).

Trichodesmium bloom distributions estimated by TRICHOSAT for the WP are shown as composites over 12 years for each of the three seasons (winter, interseason, and summer) in Fig. 6. The increase in selected Trichodesmium pixels from austral winter (Fig. 6a) to the interseason (Fig. 6b) to austral summer (Fig. 6c) in the SP is visually evident. Although there is a relatively high response of TRICHOSAT between 1998 and 1999 during boreal winter in the northern Pacific (November-December 1998 (in yellow); January-March 1999 (in black)) around $160^{\circ} \mathrm{W}$, no Trichodesmium pixels were retrieved during the same time of year between 2000-2010.

Figure 7a shows the temporal evolution of the percentage of Trichodesmium bloom pixels, the number of Trichodesmium bloom pixels divided by the total number of valid (non-cloudy) pixels x 100, identified by TRICHOSAT on a monthly basis. The algorithm was applied to the WP and to the SP domains. Regular peaks were observed from December-February nearly every year over the 12-year period examined, albeit the monthly maximum percentage of retrieved Trichodesmium pixels exhibited strong inter-annual variations. For the SP box, the percentage was above $0.2 \%$ in 1999 and in 2004-2005 and dropped to $0.01 \%$ in winter. At 


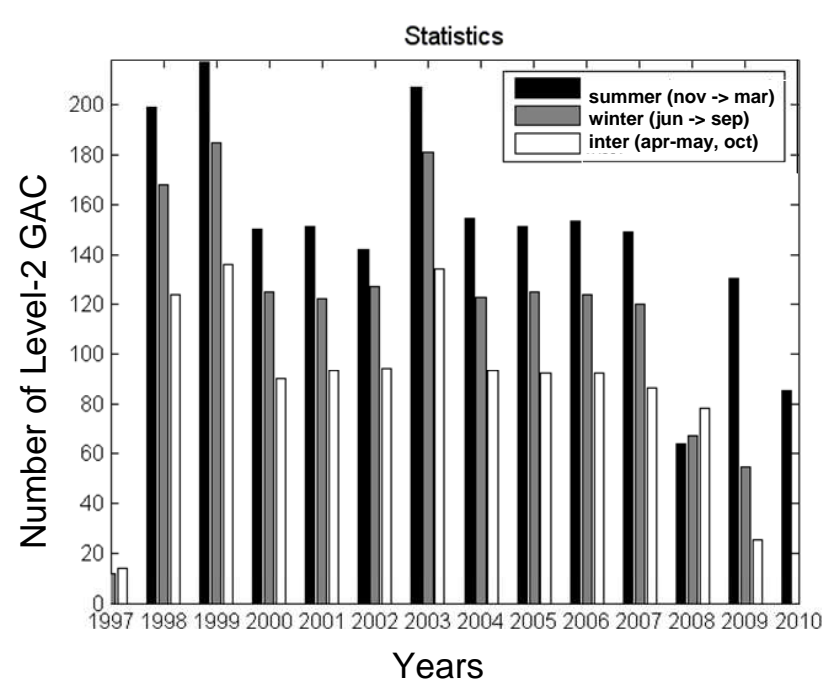

Fig. 5. Statistics of the number of SeaWiFS Level2 GAC images used to determine the spatio-temporal distribution of Trichodesmium accumulations in the SP between 1997 and 2010 by year and season. Seasons are defined as: austral summer (November to March); austral winter (June to September); interseason (April-May and October).

the monthly timescale, this fraction represents a total number of Trichodesmium bloom pixels varying from 31 to 62 in winter and from 193 to 1652 in summer (maximum in January 1999) compared to a number of valid (non-cloudy) pixels varying between $200 \times 10^{3}$ and $10^{6}$ pixels. This is equivalent to monthly surface areas varying between 500 to $1000 \mathrm{~km}^{2}$ in winter and from 3100 to $10890 \mathrm{~km}^{2}$ in summer (with a maximum of $26432 \mathrm{~km}^{2}$ in January 1999). For the larger WP box, the percentage was much reduced with a maximum of $0.03 \%$, because there were much less Trichodesmium pixels detected in the northern half of the WP domain. The mean monthly chlorophyll concentration for the SP is also shown. Note that only a few Trichodesmium peaks correspond to a secondary chlorophyll maximum, which further illustrates the independence of bloom occurrence and chlorophyll concentration.

The mean seasonal cycle of Trichodesmium bloom coverage in the SP for the 1997-2010 period is shown in Fig. 8. Over 12 years, Trichodesmium bloom maxima were observed in January and February with relatively high inter-annual variation. Similarly, the 12 -y average monthly percentage of slick observations (from Table 1, and Moutin et al., 2005 for observations before 2004), peaks between January and February.

TRICHOSAT was also applied to single daily Level2 GAC images around New Caledonia and Vanuatu and for short periods representative of summer and winter months (Fig. 9). From the 10 to the 22 of February 2003, TRICHOSAT selected pixels spread out between Vanuatu and the northwestern part of New Caledonia and Fiji (Fig. 9a).
Two observations from the French Navy coincide with TRICHOSAT pixels. From the 9 to the 15 of June 2003, no pixels were detected around New Caledonia (Fig. 9b). The few Trichodesmium pixels at the southern limit of the equatorial upwelling between $7^{\circ} \mathrm{S}$ and $5^{\circ} \mathrm{S}$ could correspond to observations of Trichodesmium blooms in the southern and northern convergence zones of the upwelling (Lebouteiller et al., 1992; Blanchot, personal communication, 2004).

The comparison between in situ observations of slicks (summed by month from Table 1) and the percentage of Trichodesmium bloom pixels detected on each single level2 GAC by TRICHOSAT in the SP for the total 1998-2010 period is shown in Fig. 10. The striking coincidence between the number of observations of surface slicks (from 1 to 6 between October 2003 and February 2004) and the peak of percentage of pixels screened by TRICHOSAT is the result of favorable observation conditions in the SP. Despite the reduced number of observations during the 2003-2004 period, the maximum percentage of Trichodesmium bloom pixels generally corresponds to a significant number $(>2)$ of observed slicks (Fig. 10).

\section{Discussion}

\subsection{General considerations of the algorithm}

\subsubsection{A rather linear RAS spectrum}

TRICHOSAT identifies pixels for which the RAS is characterized by a narrow range of $S$ (small negative numbers) and of Yt (values near 1) which implies a relatively linear RAS spectrum compared with the total range of $\mathrm{S}$ and $\mathrm{Yt}$ found within the whole Pacific Ocean. Shape criteria (bumps and troughs) of the RAS are also essential as the intersection between shape and RAS quantitative criteria is required for the successful selection of Trichodesmium bloom pixels (Fig. 4b, $\mathrm{c}, \mathrm{d})$. This means that the RAS spectra of Trichodesmium blooms are not so different to what is expected at a given chlorophyll concentration. Westberry et al. (2005) also found remarkably similar bulk reflectance spectra for cases which contained moderate amounts of Trichodesmium compared to those where it was absent. The RAS though differ from those of other phytoplankton groups defined by the PHYSAT approach (Alvain et al., 2005).

\subsubsection{A rather weak relationship with biomass}

The RAS is by definition, independent of chlorophyll concentration (second order anomaly). Therefore, it is not surprising that selected pixels correspond to a large range of Chl- $a$ values, from 0.07 to $0.3 \mathrm{mg} \mathrm{m}^{-3}$ in a single Level 2 GAC image. It has already been shown that chlorophyll associated with surface accumulations of Trichodesmium is highly variable. Also, underestimation of chlorophyll in the presence of Trichodesmium by SeaWiFS due to a strong 
a

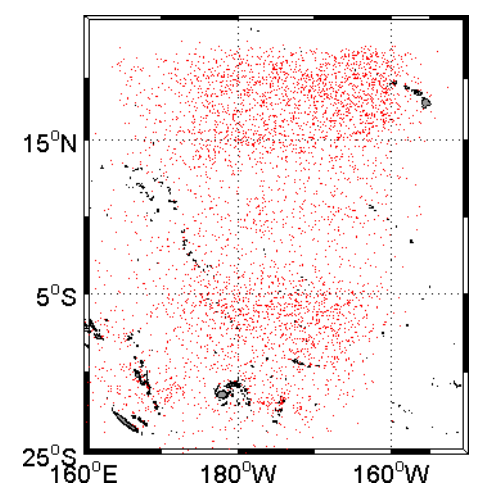

b

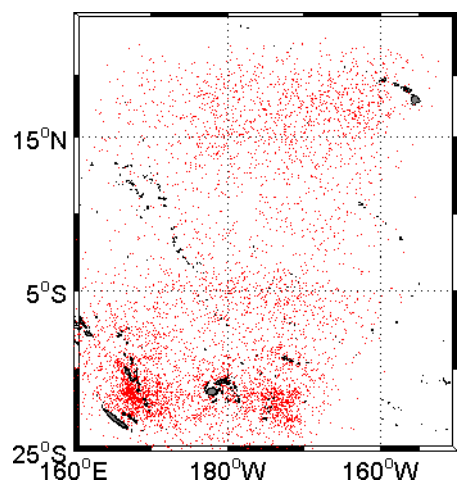

C

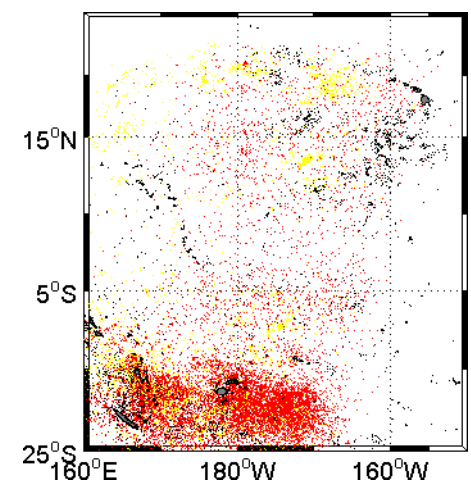

Fig. 6. Application of the TRICHOSAT algorithm to satellite data (1997 to 2010) over the large box corresponding to the Western Pacific Ocean (WP: $25^{\circ} \mathrm{N}-25^{\circ} \mathrm{S} / 160^{\circ} \mathrm{E}-160^{\circ} \mathrm{W}$ ); (a) austral winter (June to September); (b) interseason (April-May and October); (c) austral summer (November to March). The main islands of New Caledonia at $20^{\circ} \mathrm{S}$, Vanuatu at $15^{\circ} \mathrm{S}$, Fiji Islands at $17^{\circ} \mathrm{S}, \mathrm{Hawaii}^{\mathrm{I}}$ Ilands at $20^{\circ} \mathrm{N}$ are indicated in grey.
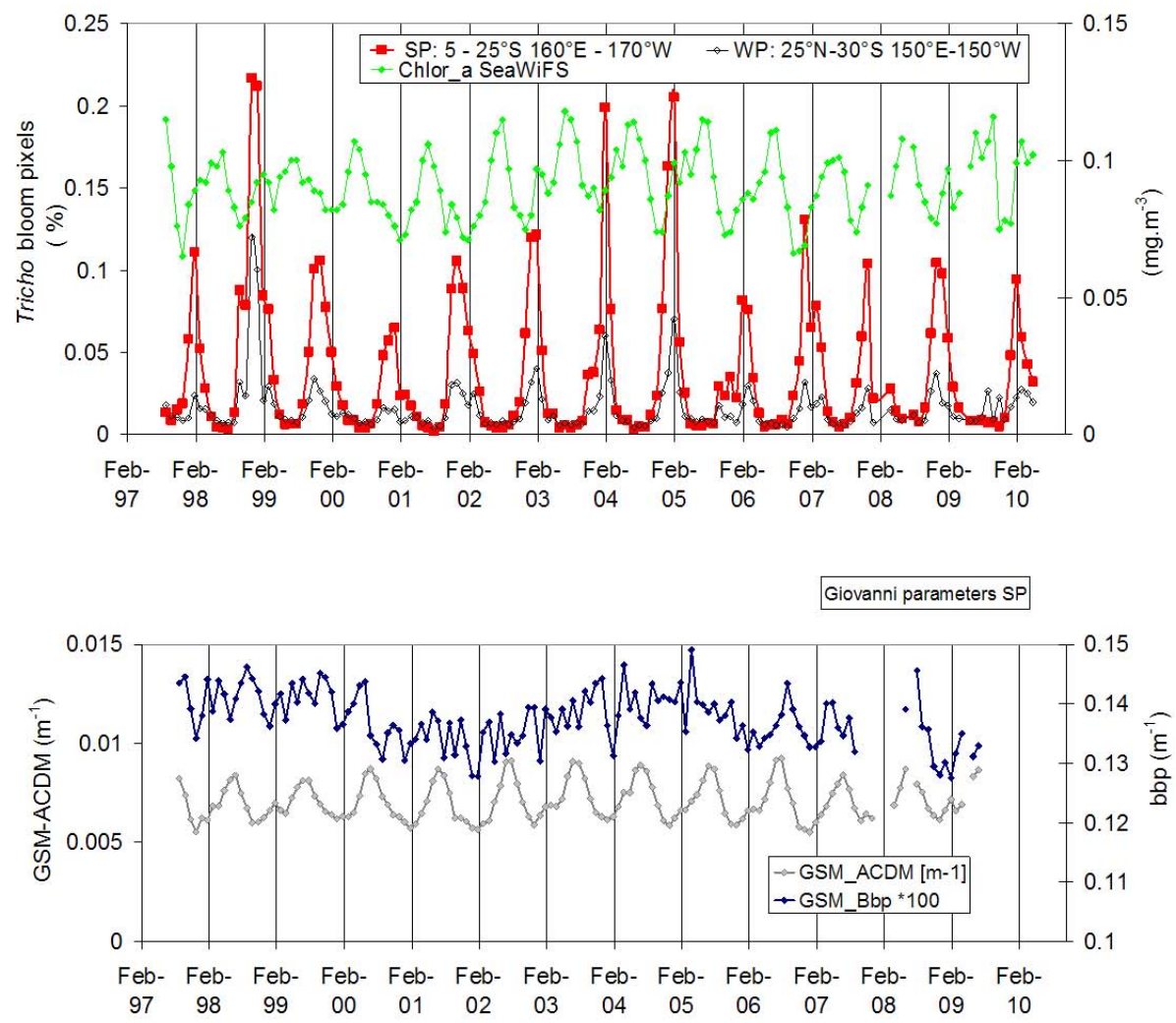

Time (Month-Year)

Fig. 7. (a) Monthly percentage of valid pixels identified as Trichodesmium when applying the TRICHOSAT algorithm to SeaWiFS Level2 imagery $(4 \mathrm{~km} \times 4 \mathrm{~km})$. Results are shown for the Southern Pacific Ocean box (SP: $\left.5^{\circ} \mathrm{S}-25^{\circ} \mathrm{S}, 150^{\circ} \mathrm{E}-190^{\circ} \mathrm{E} / 170^{\circ} \mathrm{W}\right)$ in red and for the WP (WP: $25^{\circ} \mathrm{N}-25^{\circ} \mathrm{S} / 160^{\circ} \mathrm{E}-160^{\circ} \mathrm{W}$ ) as black. Trichodesmium blooms occur regularly during the austral summer and peak in February. SeaWiFS chlorophyll-a for SP also shown as green line (note that the monthly chlorophyll is smoothed compared to chlorophyll concentrations in pixels of individual level2-GAC images) (b) GSM-derived ACDM absorption coefficient ( $\mathrm{m}^{-1}$ ) (grey line) and scaled particulate backscattering coefficient $\left(\mathrm{m}^{-1}\right)$ (dark blue line). 
a
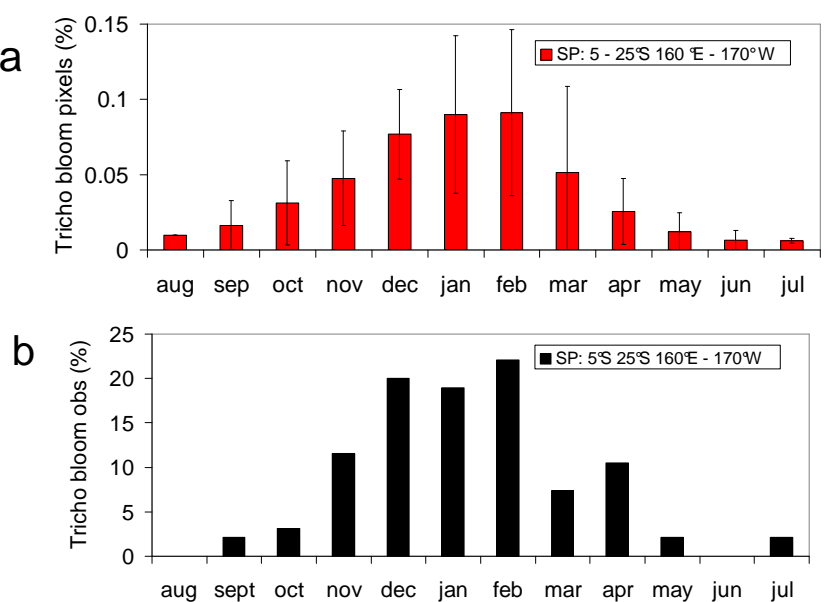

Fig. 8. (a) Histogram representing the mean annual cycle of percentage Trichodesmium bloom pixels $( \pm 1 \sigma)$ over the 12 years (1998-2010) of the SeaWiFS acquisition period; (b) Histogram representing the mean annual number of in situ slick observations $(\mathrm{Nb}$, from Table 1).

package effect in filaments and colonies has been observed (Subramaniam et al., 1999; Dupouy et al., 2008). As TRICHOSAT probably screens pixels containing high Trichodesmium concentrations mixed in the upper oceanic layer or forming surface accumulations, this might introduce scatter to the relationship between reflectance and chlorophyll concentration.

\subsubsection{Comparison with previous algorithms}

The percentage of TRICHOSAT selected pixels in the New Caledonia region (Figs. 7, 8, 10) is low (maximum $0.2 \%$ of total valid pixels). However, TRICHOSAT was designed to detect a specific case of Trichodesmium bloom when it is concentrated within a few $\mathrm{mm}$ of the sea surface and visible by eye. Under these circumstances, densities are approximately between 17000 trichomes. $\mathrm{L}^{-1}$ and $3910^{6}$ trichomes. $L^{-1}$ (Devassy et al., 1978). Assuming an accumulation which contains $\sim 3 \mathrm{~g} \mathrm{~m}^{-3}$ Chl- $a$ distributed over a $1 \mathrm{~mm}$ thickness, this is equivalent to an integrated concentration of $3 \mathrm{mg} \mathrm{m}^{-2}$ of Chl- $a$ just due to Trichodesmium. If the bloom occupies an area of $100 \mathrm{~m}^{2}$, i.e. the $1 / 160000$ of the pixel size, the mean Chl- $a$ concentration linked to Trichodesmium in the pixel is $0.018 \mathrm{mg} \mathrm{m}^{-2}$. Supposing that the rest of the phytoplankton represents on average $0.1 \mathrm{mg} \mathrm{m}^{-3}$, we need about $500 \mathrm{~m}^{2}$ of concentrated accumulation within the pixel to double the chlorophyll concentration. The probability of finding Trichodesmium bloom pixels is low to begin with, much less to observe a $500 \mathrm{~m}^{2}$ concentrated patch within a pixel. Nevertheless, the algorithms of Subramaniam et al. (2002) and Westberry et al. (2005) (hereafter denoted as W05) detect even fewer pixels (100 times less). In the SP, the W05 algorithm retrieves a majority of highly reflective
TRICHOSAT/W05, 10-22 Feb. 2003
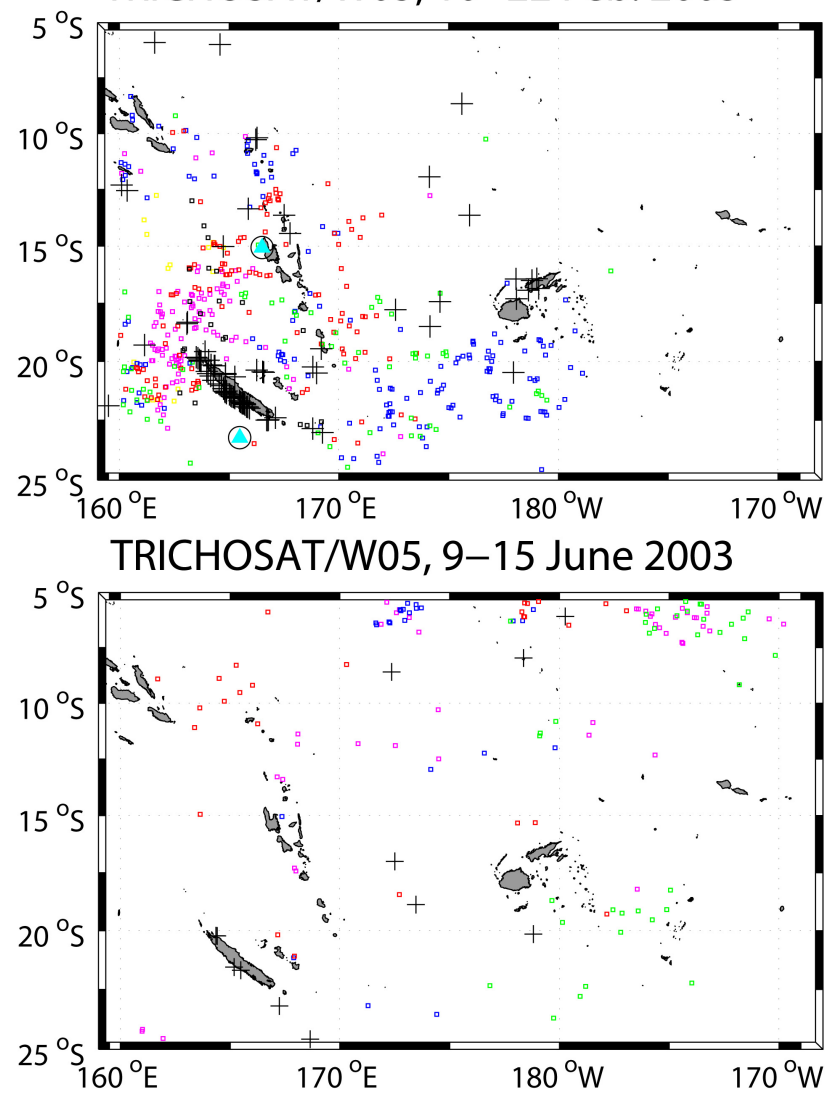

Fig. 9. Spatial distributions of Trichodesmium bloom pixels retrieved with TRICHOSAT in the SP box $\left(5^{\circ} \mathrm{S}-25^{\circ} \mathrm{S} 160^{\circ}-170^{\circ} \mathrm{W}\right)$ for short periods (a) during Austral summer, 10-22 February 2003 (In Yellow: 9 February, in Magenta: 11-12 February, in Blue: 1314 February, in Red : 16-17 February, in Green: 18-19 February, in Black: 21 February) and (b) during Austral winter, 9-15 June, 2003 (In Magenta: 9 June, in Blue: 11 June, in Red: 13 June, in Green: 15 June). The two aerial observations from Table 1 are added. Each pixel represents a surface of $16 \mathrm{~km}^{2}$ (the size of colored squares is equivalent to a surface of $256 \mathrm{~km}^{2}$, i.e. 16 "real" SeaWifs pixels of $4 \times 4 \mathrm{~km}$ ). Also shown are results obtained from Westberry et al. (2005) (black crosses) for the same time periods. In grey, the main lands of New Caledonia at $20^{\circ} \mathrm{S}$, Vanuatu at $15^{\circ} \mathrm{S}$ and Fiji Islands at $17^{\circ} \mathrm{S}$.

pixels, most of which corresponding to extremely high reflectance of New Caledonia or Fijian sandy lagoons that are later masked as shallow waters. Furthermore, only 15 points are identified as Trichodesmium by W05 and there is no indication of seasonal variation. Recall that the W05 algorithm was built to detect Trichodesmium at bloom concentrations (Chl- $a$ threshold for a bloom was set to $0.8 \mathrm{mg} \mathrm{m}^{-3}$ ) while TRICHOSAT detection does not imply Chl- $a$ concentration threshold. The rather weak spectral anomalies of Trichodesmium pixels may explain why W05 does not detect more blooms in the SP. The first published set of criteria for screening pixels containing Trichodesmium overemphasized 


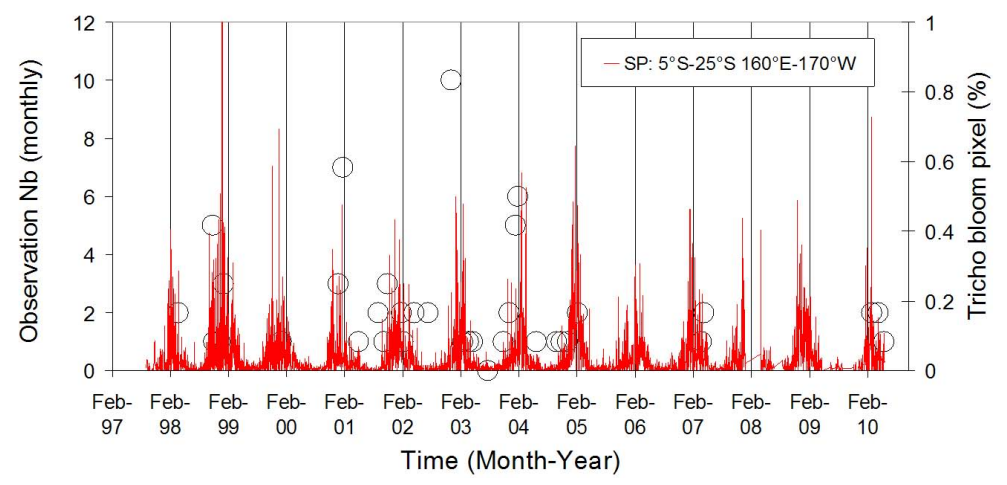

Fig. 10. Daily evolution of the TRICHOSAT retrievals shown as red bars (in \% pixels, over the SP domain) from individual SeaWiFS Level 2 GAC images, and number of surface slicks observed per month ( $\mathrm{Nb}$ issued from Table 1) as black open circles in the SP area for the period 1998-2010.

backscattering (for $\lambda>500 \mathrm{~nm}$ ) and Colored Dissolved Organic Matter (CDOM) absorption (for $\lambda<440 \mathrm{~nm}$ ) so that finally a refined model using subtle variations in reflectances between phytoplankton and Trichodesmium spectra had to be used (Westberry and Siegel, 2006). In the SP, Trichodesmium bloom signatures may not be strong enough to be detected by W05, or blooms occur in filaments whose signatures may not be strong enough to affect $16 \mathrm{~km}^{2}$ pixels. Finally, living colonies of Trichodesmium are often associated with other phytoplankton as observed during the Diapalis 07 cruise (Tenório, 2006) and serve to create a mixed optical signal obfuscating detection. Trichodesmium biomass could then be well below the W05 detection threshold, and/or the other phytoplankton biomass would be higher in proportion in the SP than in the northern hemisphere. This may also explain why the W05 algorithm detects similar Trichodesmium pixel numbers in winter and in summer. Overall, TRICHOSAT was tuned to detect the Trichodesmium blooms that we have observed in the field in summer, and the W05 algorithm fails to detect these blooms.

\subsection{Optical validation of the algorithm}

Ideally, one would validate the RAS of TRICHOSAT detected Trichodesmium pixels with in situ radiometric measurements made in Trichodesmium surface blooms. Unfortunately, such a task is difficult as there is no direct measurement of the RAS which would require accurate measurements of above in water reflectance. Past work has recognized this as problematic (Subramaniam et al., 1999; Kutzer, 2009). Recall that the Trichodesmium bloom RAS is defined by a very small range of S and Yt. This implies that SeaWiFS spectra are not so different from what they are expected at a given $\mathrm{K}_{490}$ (i.e. at a given Chl- $a$ concentration) despite the fact that filamentous cyanobacteria blooms appear brighter than surrounding water areas due to high backscattering associated with gas vesicle and to a specific microbiotope (bacteria, detritus) (Subramaniam et al., 1999).
One of the TRICHOSAT shape criterion is that the RAS must never show a bump at $555 \mathrm{~nm}$. It is well known that reflectance spectra of colonies, assembled on filters (Dupouy et al., 2008) are characterized by a succession of troughs, each trough corresponding to different pigment absorption maxima (e.g., chlorophyll, phycourobilin and phycoerythrobilin). A trough around $550-560 \mathrm{~nm}$ related to phycoerythrin is systematically observed in collected Trichodesmium suspension (PSICAM absorption measurements, Dupouy and Röttgers, 2010; raw H6-backscattering spectra, Dupouy et al., 2008), and in situ samples during the 2004 bloom (unp. data).

The second robust criterion is that the RAS must never show a trough at $670 \mathrm{~nm}$ (rather a bump at $670 \mathrm{~nm}$ ). It has been shown that high near-infrared reflectance is observed if colonies are accumulated on top of the water (Subramaniam et al., 1999; Dupouy et al., 2008). The RAS would then depend on the proportion of colonies lying above the water surface, its thickness, the physiological characteristics of colonies, and the age of the bloom (Dupouy et al., 1990, 1992, 2008). The high reflectance of blooms at $670 \mathrm{~nm}$ which was observed with the Coastal Zone Colour Scanner (CZCS) could provide an approach to detecting slicks from MODIS (Hu et al., 2010; McKinna et al., 2011) or IRS-P4 OCM (Sarangi et al., 2004).

There was no robust criteria for the RAS at $412 \mathrm{~nm}$ even though a significant RAS feature was expected at this wavelength as it has been shown that Dissolved Organic Carbon (DOC) and CDOM is released from dead or living colonies (Subramaniam et al., 1999). Optical characteristics of surrounding waters may also be important for defining RAS of Trichodesmium blooms. As seen in Fig. 7b, the dissolved matter + detritus absorption coefficient, ACDM (derived from the GSM optical inversion model at $443 \mathrm{~nm}$; Maritorena et al, 2002) for the SP box experiences a strong seasonal variation due to the well documented annual cycle of solar bleaching and photolysis in the South Pacific (Siegel et al., 2002, 2005). Trichodesmium surface blooms correspond to 
the period of minimum CDOM concentration in summer. In the SP, some peaks are associated with a secondary CDOM maximum which could be produced by blooms (e.g., February 1999, 2003, 2004 and 2006). In contrast, the particulate backscattering coefficient, $b_{\mathrm{bp}}$ (also derived from Maritorena et al., 2002) was rather constant though noisy over the 12 year period (Fig. 7b). Trichodesmium blooms correspond to the period of minimum backscattering, as a weak maximum appears in June-September (more or less in phase with chlorophyll concentration). The $b_{\mathrm{bp}}$ cycle in the SP could be linked to small-sized detritus as suggested by Loisel et al. (2006) rather than to Trichodesmium blooms.

The relatively strong response by TRICHOSAT in the northern Pacific around $160^{\circ} \mathrm{W}$ between 1998 and 1999 during boreal winter (November-December 1998 (yellow in Fig. 6); January-March 1999 (black in Fig. 6)) does not correspond to visual observations of blooms in this area (Dore et al., 2008). Further, from 2000 to 2010, no Trichodesmium pixels were retrieved during boreal winter in the Northern Pacific area by TRICHOSAT. We have no explanation for what happened during the 98-99 boreal winter period near Hawaii. We conclude that there is some signal there picked up by the TRICHOSAT algorithm. Blooms of unknown origin have been reported previously using CZCS observations in December (Dore et al., 2008). This has to be caused by floating living material similar in reflectance to Trichodesmium and therefore having the same SeaWiFS RAS. Also, there may be a different relationship between reflectance and $\mathrm{K}_{490}$ in SeaWiFS data for these five months than for the rest of the studied period. Further research should be conducted to determine the nature of this optical signal in the Northern Pacific.

\subsection{Relationships with phytoplankton in the water column}

The austral summer maxima of surface blooms detected with TRICHOSAT in SP (Fig. 7a) correspond with the interannual variations of Trichodesmium abundances in the surface layer ( $2 \mathrm{~m}$, Table 2). Lower Trichodesmium densities and slick observations were observed in situ in April-May 2002-2003 and October 2001-2003 (Tables 1 and 2) which also appear as lower surface bloom extent identified by TRICHOSAT. At the opposite, a surprisingly low Trichodesmium concentration (200-400 trichomes. $\mathrm{L}^{-1}$ ) was observed in the water column in December 2001-January 2002 while the surface bloom extent by TRICHOSAT was high. In JuneAugust 2003 (winter season), Trichodesmium was rare and total chlorophyll in the $>10 \mu \mathrm{m}$ fraction was less than $10 \%$ (Table 2). Thus, the community was dominated by picoplankton (cyanobacteria and picoeucaryotes) and the surface bloom extent detected by TRICHOSAT was minimum.

The low percentage of detected surface blooms by TRICHOSAT, occurring in summer, corresponds to a physiological state where colonies become buoyant because they cannot synthesize ballast anymore due to phosphate limitation and thus float as dead material and accumulate at the surface (Moutin et al., 2005). Such conditions may be spatially and temporally de-coupled from actively growing Trichodesmium colonies in sub-bloom concentrations (observed from 0 to $60 \mathrm{~m}$ during Diapalis cruises). Applications of the algorithm for an estimate of potential nitrogen fixation would require a better understanding of the relationship between the development of Trichodesmium over the whole water column and surface accumulations.

Last, the relative abundance of Trichodesmium and other nitrogen-fixing cyanobacteria need to be determined. Coccoid cyanobacteria (Crocosphaera, Cyanothece) have been detected from their phycoerythrin signature (Neveux et al., 2006) and flow cytometric properties in the SP (Campbell et al., 2006; Moisander et al., 2010; Sato et al., 2010; Biegala, personal communication, 2010). Nitrogen-fixing heterotrophic bacteria have also been identified in the SP (Rieman et al., 2010). During summer, nitrogen fixation both from filamentous and coccoid cyanobacteria (Garcia et al., 2007; Hynes et al., 2009) exhibited high rates (151$703 \mu \mathrm{M} \mathrm{N}_{2} \mathrm{~m}^{-2} \mathrm{~d}^{-1}$ ) compared to those measured north of Papua-New Guinea (Bonnet et al., 2009). Late 2007 field observations confirmed the dominance of Trichodesmium in association with Crocosphaera in the South Pacific, spatially decoupled from unicellular picocyanobacteria (Hewson et al., 2009; Moisander et al., 2010; Sato et al., 2010). Crocosphaera, Cyanothece and picocyanobacteria populations may form a high fraction of total nitrogen fixation which will not be detected by TRICHOSAT.

Furthermore, better knowledge of the spatial and temporal association of coccoid cyanobacteria with Trichodesmium (Campbell et al., 2006; Sato et al., 2010; Moisander et al., 2010), would help in getting a global view of all nitrogen fixing organisms.

\section{Conclusions}

The TRICHOSAT algorithm is efficient at discriminating Trichodesmium surface accumulations in the Western tropical Pacific Ocean. Its results can complement the identification of major functional groups provided by PHYSAT. TRICHOSAT was developed for the SP and is even able to follow the daily evolution of surface blooms over the whole 1998-2010 period (Fig. 10). Its application at a global scale requires that the RAS criteria in TRICHOSAT is representative of Trichodesmium blooms in other regions, and also that characteristics of surrounding waters resemble those in the SP. Reasons why detection of Trichodesmium with other algorithms was much less successful (100 times less pixels and no seasonality) in the Western Tropical Pacific Ocean may be, (1) low Trichodesmium concentrations, (2) a weak discriminating optical signal due to a mixed optical assemblage, and (3) a significantly different Trichodesmium optical signature in the SP than other areas. 
Trichodesmium experienced large inter-annual variability in the region with maxima in 1999, 2003, 2004 and 2005, while 2001 and 2002 were less favourable. This inter-annual variability might be related to large scale circulation dynamics. For example, the bifurcation latitude of the South Equatorial Current is found to move southward from about $15^{\circ} \mathrm{S}$ near the surface to south of $22^{\circ} \mathrm{S}$ in the intermediate layers ( $\mathrm{Qu}$ and Lindstrom, 2002) associated with large scale changes in the phosphate pool (Dyrhman et al., 2006; Tadokoro et al., 2009). Also, iron-rich dust deposition patterns (Gao et al., 2001) may be highly variable in time. Whether other phytoplankton blooms are triggered by Trichodesmium decomposition of organic matter is also of interest (Chen et al., 2008, 2011). Late summer chlorophyll blooms in the oligotrophic North Pacific subtropical gyre may be fuelled by Trichodesmium (Wilson and Qiu, 2008).

TRICHOSAT demonstrated that Trichodesmium blooms are a common feature in the SP during austral summer (October to March). They are temporally and spatially linked to diverse nitrogen-fixing populations and can be identified as a major potential carbon sink in the SP. A large-scale physical and biogeochemical modeling of the distribution of Trichodesmium is required in the SP.

Acknowledgements. The authors are grateful to all Commandants of the French Navy in New Caledonia over the 1998-2010 period for aerial and ship observations, and Commandants of Vessels " $\mathrm{La}$ Glorieuse", "La Moqueuse”, and the Batral "Jacques Cartier" as well as the French Navy based at La Tontouta Airport aeronaval basis for airplane observations. We also thank Raymond Proner, Hervé Le Houarno, Jean-François Barazer, as well as the crew of the R/V Alis for their shipboard support during the operations at sea and observations. We thank Guillaume Dirberg for participation in the MOTEVAS cruise and for photography at the microscope in the laboratory of our appreciated and regretted colleague Guy Cabioch (LOCEAN, Paris) allowing identifications of morphotypes. Thanks to Alain Lapetite for his help in collecting sea samples and to all colleagues, Martine Rodier, Jean-Yves Panché, and Francis Gallois, Jean-Louis Laurent, Michel Lardy, Stéphane Calmant, Jean-Michel Boré, students Xavier Combres, Romain Charraudeau, Philippe Borsa, Céline Chauvin, and Guillaume Dirberg for their kindly help during transects. Thanks to Marcio Tenório for biomass measurements and cell counts during the Diapalis cruises. This work would not have been possible without the constant support by Aubert Le Bouteiller, Chief scientist of the Diapalis cruises on the R/V Alis and of the DIAPAZON (DIAzotrophy PAcific ZONe, 2001-2003) program. Finally, we thank NASA for the MODIS real-time observation (Acker and Lepthouck, 2007) web site http://earthobservatory.nasa.gov/IOTD/view.php?id=46954 and Image Science and Analysis Laboratory, NASA-Johnson Space Center (The Gateway to Astronaut Photography of Earth). Analyses and visualizations for Chl- $a$ used in this paper were produced with the Giovanni online data system, developed and maintained by the NASA GES DISC. "Giovanni”, an easy-to-use, Web-based interface for the visualization and analysis of Earth Science data provided by the GES DISC DAAC, the data used in this effort were acquired as part of the activities of NASA's Science Mission
Directorate, and are archived and distributed by the Goddard Earth Sciences (GES) Data and Information Services Center (DISC).

This work was supported by the Institut de Recherche pour le Développement (IRD), the Institut National des Sciences de l'Univers (INSU) and by the French program PROOF (PROcessus biogéochimiques dans l'Océan et Flux). We thank the Ocean Biology Processing Group "Sea-viewing Wide Field-of-view Sensor Project" and particularly Gene Feldman at the Goddard Space Flight Center of NASA (USA).

Edited by: G. Herndl

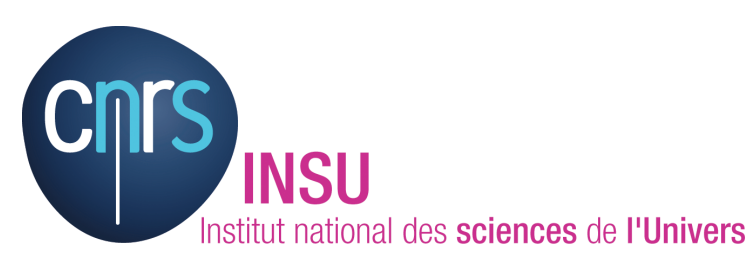

The publication of this article is financed by CNRS-INSU.

\section{References}

Acker, J. G. and Leptoukh, G.: Online Analysis Enhances Use of NASA Earth Science Data, Eos, Trans. AGU, 88, 14, doi:10.1029/2007EO020003, 2007.

Alvain, S., Moulin, C., Dandonneau, Y., and Breon, F. M.: Remote sensing of phytoplankton group in case 1 waters from global SeaWiFS imagery, Deep-Sea Res. Pt. I, 52, 1989-2004, 2005.

Barcelos e Ramos, J., Biswas, H., Schulz, K. G., LaRoche, J., and Riebesell, U.: Effect of rising atmospheric carbon dioxide on the marine nitrogen fixer Trichodesmium, Global Biogeochem. Cy., 21, GB2028, doi:10.1029/2006GB002898, 2007.

Bell, P. R. F., Elmetri, I., and Uwins, P.: Nitrogen fixation by Trichodesmium spp. in the Central and Northern Great Barrier Reef Lagoon: relative importance of the fixed-nitrogen load, Mar. Ecol.-Prog. Ser., 186, 119-126, 1999.

Berman-Frank, I., Bidle, K. D., Haramaty, L., and Falkowski, P. G.: The demise of the marine cyanobacterium, Trichodesmium spp., via an autocatalyzed cell death pathway, Limnol. Oceanogr., 49, 997-1005, 2004.

Bonnet, S., Biegala, I. C., Dutrieux, P., Slemons, L. O., and Capone, D. G.: Nitrogen fixation in the western equatorial Pacific: rates, diazotrophic cyanobacterial size class distribution, and biogeochemical significance, Global Biogeochem. Cy., 23, 1-13, 2009.

Bowman, T. E. and Lancaster, L. J.: A bloom of the planktonic blue-green alga, Trichodesmium erythraeum, in the Tonga Islands, Limnol. Oceanogr., 10, 291-293, 1965.

Bracher, A., Vountas, M., Dinter, T., Burrows, J. P., Röttgers, R., and Peeken, I.: Quantitative observation of cyanobacteria and diatoms from space using PhytoDOAS on SCIAMACHY data, Biogeosciences, 6, 751-764, doi:10.5194/bg-6-751-2009, 2009.

Breitbarth, E., Oschlies, A., and LaRoche, J.: Physiological constraints on the global distribution of Trichodesmium effect of temperature on diazotrophy, Biogeosciences, 4, 53-61, doi:10.5194/bg-4-53-2007, 2007. 
Bryan, S. E., Cook, A., Evans, J. P., Colls, P. W., Wells, M. G., Lawrence, M. G., Jell, J. S., Greig, A., and Leslie, E.: Pumice rafting and faunal dispersion during the 2001-2002 in the Southwest Pacific: record of a dacidic submarine explosive eruption from Tonga, Earth Plan. Sci. Lett., 227, 135-154, 2004.

Campbell, L., Carpenter, E. J., Montoya, J. P., Kustka, A. B., and Capone, D. G.: Picoplankton community structure within and outside a Trichodesmium bloom in the southwestern Pacific Ocean, Vie Milieu, 55, 185-195, 2006.

Capone, D. G. and Knapp, A. N.: Oceanography, A marine nitrogen cycle fix? Nature, 445, 159-160, doi:10.1038/445159A, 2007.

Capone, D. G., Burns, J. A., Montoya, J. P., Subramaniam, A., Mahaffey, C., Gunderson, T., Michaels, A. F., and Carpenter E. J.: Nitrogen fixation by Trichodesmium spp.: an important source of new nitrogen to the tropical and subtropical North Atlantic Ocean. Global Biogeochem. Cy., 19, GB2024, doi:10.1029/2004GB002331, 2005.

Capone, D. G., Zehr, J. P., Paerl, H. W. , Berman, B., and Carpenter, E. J.: Trichodesmium, a globally significant marine cyanobacterium, Science, 276, 1221-1229, 1997.

Carpenter, E. J.: Nitrogen fixation by marine Oscillatoria Trichodesmium in the world's ocean, in: Nitrogen in the Marine Environment, edited by: Carpenter, E. J. and Capone, D. J., Academic Press, New-York, 65-103, 1983.

Carpenter, E. J., O’Neil, J. M., Dawson, R., Capone, D. G., Siddiqui, P. J. A., Roenneberg, T., and Bergman, B.: The tropical diazotrophic phytoplankter Trichodesmium: biological characteristics of two common species, Mar. Ecol.-Prog. Ser., 95, 295-304, 1993.

Chen, Y. L. L., Chen, H. Y., Tuo, S., and Ohki, K.: Seasonal dynamics of new production from Trichodesmium $\mathrm{N}_{2}$ fixation and nitrate uptake in the upstream Kuroshio and South China Sea basin, Limnol. Oceanogr., 53, 1705-1721, 2008.

Chen, Y. L. L., Tuo, S., and Chen, H. Y.: Co-occurrence and transfer of fixed nitrogen from Trichodesmium spp. to diatoms in the lowlatitude Kuroshio Current in the NW Pacific, Mar. Ecol.-Prog. Ser., 421, 25-38, 2011.

Church, M. J., Bjorkman, K. M., Karl, D. M., Saito, M. A., and Zehr, J. P.: Regional distributions of nitrogen fixing bacteria in the Pacific Ocean, Limnol. Oceanogr., 53, 63-77, 2008.

Church, M. J., Mahaffey, C., Letelier, R. M., Lukas, R., Zehr, J. P., and Karl, D. M.: Physical forcing of nitrogen fixation and diazotroph community structure in the North Pacific Subtropical Gyre, Global Biogeochem. Cy., 23, GB2020, doi:10.1029/2008GB003418, 2009.

Dandonneau, Y. and Gohin, F.: Meridional and seasonal variations of the sea surface chlorophyll concentration in the South western tropical Pacific ocean, Deep-Sea Res., 31, 137-139, 1984.

Deutsch, C., Sarmiento, J. L., Sigman, D. M., Gruber, N., and Dunne, J. P.: Spatial coupling of nitrogen inputs and losses in the ocean, Nature, 445, 159-160, doi:10.1038/nature05392, 2007.

Devassy, V. P., Bhattathiri, P. M. A., and Qasim, S. Z.: Trichodesmium phenomenon, Ind. J. Mar. Sci., 7, 168-186, 1978.

Dore, J. E., Letelier, R. M., Church, M. J., Lukas, R., and Karl, D. M.: Summer phytoplankton blooms in the oligotrophic North Pacific Subtropical gyre: Historical perspective and recent observations, Prog. Oceanogr., 76, 2-38, 2008.
Dupouy, C.: La chlorophylle de surface observée par le satellite NIMBUS-7 CZCS autour de la Nouvelle Calédonie et de ses dépendances. Une première analyse. Bulletin de l'Institut Océanographique de Monaco, 125-148. Colloque Scientifique Franco-Japonais; Colloque d'Océanographie, 5, 2, Tokyo; Shimizu J. P. N, 3-13 October 1998, 1990.

Dupouy, C.: Discoloured waters in the Melanesian archipelago New Caledonia and Vanuatu. The value of the Nimbus-7 Coastal Zone Colour Scanner observations, in: Marine Pelagic Cyanobacteria: Trichodesmium and other diazotrophs, edited by: Carpenter, E. J., Capone, D. G. and Rueter J. G., Kluwer Academic Press, NATO Adv. Sci. I. C-Mat., 362, 177-191, 1992.

Dupouy, C. and Röttgers, R.: Absorption by different components during a high freshwater event of the 2008 La Nina episode in a tropical lagoon, Poster Session "Bio-optics and biogeochemistry”, Ocean Optics XX, Anchorage (Alaska), 25-30 september 2010.

Dupouy, C., Petit, M., and Dandonneau, Y.: Satellite detected cyanobacteria bloom in the southwestern tropical Pacific. Implication for oceanic nitrogen fixation, Int. J. Rem. Sens., 9, 389396, 1988.

Dupouy, C., Neveux, J., Subramaniam, A., Mulholland, M., Campbell, L., Montoya, J., Carpenter, E., and Capone, D.: Satellite captures Trichodesmium blooms in the Southwestern Tropical Pacific, EOS Trans. AGU, 81, 13, doi:10.1029/00EO00008, 2000.

Dupouy, C., Dirgerg, G., Tenório, M. M. B., Neveux, J., and Le Bouteiller, A.: Surveillance des Trichodesmium autour de la Nouvelle-Calédonie, du Vanuatu, de Fidji et de Tonga 19982004, Archives Sciences de la Mer, 7, 51, 2004a.

Dupouy, C., Neveux, J., and Le Bouteiller, A.: Spatial and temporal analysis of SeaWIFS sea surface chlorophyll, temperature, winds and sea level anomalies in the South Tropical Pacific Ocean $\left(10^{\circ} \mathrm{S}-25^{\circ} \mathrm{S}, 150^{\circ} \mathrm{E}-180^{\circ} \mathrm{E}\right)$, in: Proceedings "6ème conférence PORSEC, Pan Ocean Remote Sensing Conference”, 29 November-3 December 2004, Gayana, Conception (Chili), Gayana 68(2) Suppl. I. Proc. 161-166, 2004 ISSN 0717-652X, doi:10.4067/S0717-65382004000200030, 2004b.

Dupouy, C., Neveux J., Dirberg, G., Röttgers, R., Tenório, M. M. B., and Ouillon, S.: Bio-optical properties of the marine cyanobacteria Trichodesmium spp., J. Appl. Remote Sens., 2, 117. doi:10.1117/1.2839036, 2008.

Dyrhman, S. T., Chappell, P. D., Haley, S. T., Moffett, J. W., Orchard, E. D., Waterbury, J. B., and Webb, E. A.: Phosphonate utilization by the globally important marine diazotroph Trichodesmium, Nature, 439, 68-71, 2006.

Falkowski, P. G.: Evolution of the nitrogen cycle and its influence on the biological sequestration of $\mathrm{CO}_{2}$ in the ocean, Nature, 387, 272-275, 1997.

Feldman, G. C. and McClain C. R.: Ocean Color Web, edited by: Kuring, N., Bailey, S. W., Franz, B. F., Meister, G., Werdell, P. J., and Eplee, R. E., NASA Goddard Space Flight Center, 2010.

Furnas, M. J.: Cyclonic disturbance and a phytoplankton bloom in a tropical shelf ecosystem, in: Red tides: Environmental Science and Toxicology, edited by: Okaichi, T., Anderson, D. M., and Nemoto, T., Elsevier, Amsterdam, 271-274, 1989. 
Gao, Y., Kaufman, Y. J., Tanre, D., Kolber, D., and Falkowski, P.: Seasonal distributions of aeolian iron fluxes to the global ocean, Geophys. Res. Lett., 28, 29-32, 2001.

Garcia, N., Raimbault, P., and Sandroni, V.: Seasonal nitrogen fixation and primary production in the southwest Pacific: nanoplankton diazotrophy and transfer of nitrogen to picoplankton organisms, Mar. Ecol.-Prog. Ser. 343, 25-33, 2007.

Gruber, N. and Sarmiento, J. L.: Global patterns of marine nitrogen fixation and denitrification, Global Biogeochem. Cy., 11, 235266, 1997.

Hashihama, F., Sato, M., Takeda, S., Kanda, J., and Furuya, K.: Mesoscale decrease of surface phosphate and associated phytoplanktonic dynamics in the vicinity of the subtropical South Pacific Islands, Deep-Sea Res. Pt. I, 57, 338-350, 2010.

Hewson, I., Poretsky, R. S., Dyhrman, S. T., Zielinski, B., White, A. E., Tripp, H. J., Montoya., J., and Zehr, J. P.: Microbial community gene expression within colonies of the diazotroph, Trichodesmium, from the Southwest Pacific Ocean, ISME, 1-15, 2009.

Hu, C., Cannizzaro, J., Carder, K. L., Muller-Karger, F. E., and Hardy, R.: Remote detection of Trichodesmium blooms in optically complex coastal waters: Examples with MODIS fullspectral data, Remote Sens. Environ., 114, 2048-2058, 2010.

Hutchins, D. A., Fu, F.-X., Zhang, Y., Warner, M. E., Portune, K., Bernhardt, P. W., and Mulholland, M. R.: CO2 control of Trichodesmium $\mathrm{N}_{2}$ fixation, photosynthesis, growth rates, and elemental ratios: implications for past, present, and future ocean biogeochemistry, Limnol. Oceanogr., 52, 1293-1304, 2007.

Hynes, A. M., Chappell, P. D., Dyhrman, S. T., Doney, S. C., and Webb, E. A.: Cross-basin comparison of phosphorus stress and nitrogen fixation in Trichodesmium, Limnol. Oceanogr., 54, 5, 1438-1448, 2009.

Karl, D. M., Michaels, A., Bergman, B., Capone, D., Carpenter, E., Letelier, R., Lipschultz, F., Paerl, H., Sigman, D., and Stal, L.: Dinitrogen fixation in the world's oceans, Biogeochemistry, 57/58, 47-98, 2002.

Kitajima, S., Furuya, K., Hashihama, F., and Takeda, S.: Latitudinal distribution of diazotrophs and their nitrogen fixation in the tropical and subtropical western North Pacific, Limnol. Oceanogr., 54, 537-547, 2009.

Konno, U., Tsunogai, U., Komatsu, D. D., Daita, S., Nakagawa, F., Tsuda, A., Matsui, T., Eum, Y.-J., and Suzuki, K.: Determination of total N2 fixation rates in the ocean taking into account both the particulate and filtrate fractions, Biogeosciences, 7, 2369-2377, doi:10.5194/bg-7-2369-2010, 2010.

Kranz, S., Sültemeyer, D., Richter, K. U., and Rost, B.: Carbon acquisition by Trichodesmium: the effect of $p \mathrm{CO} 2$ and diurnal changes, Limnol. Oceanogr., 54, 548-559, 2009.

Kuchler, D. and Jupp, D. L. B.: Shuttle photograph captures massive phytoplankton bloom in the Great Barrier Reef, Int. J. Remote Sens., 9, 1299-1301, 1988.

Kutzer, T.: Passive optical remote sensing of cyanobacteria and other intense phytoplankton blooms in coastal and inland waters, Int. J. Remote Sens., 30, 4401-4425, 2009.

Lantoine, F. and Neveux, J.: Spatial and seasonal variations in abundance and spectral characteristics of phycoerythrins in the Tropical Northeastern Atlantic Ocean, Deep-Sea Res. Pt. I, 44, $223-$ 246, 1997.
LaRoche, J. and Breitbarth, E.: Importance of the diazotrophs as a source of new nitrogen in the ocean, J. Sea Res., 53, 67-69, 2005.

Levitan, O., Brown, C. M. B., Sudhaus, S., Campbell, D., LaRoche, J., and Berman-Frank, I.: Regulation of nitrogen metabolism in the marine diazotroph Trichodesmium IMS101 under varying temperatures and atmospheric CO2 concentrations, Environ. Microb., 12, 1899-1912, 2010.

Loisel, H., Nicolas, J. M., Sciandra, A., and Stramski, D.: Spectral dependency of optical backscattering by marine particles from satellite remote sensing of the global ocean”, J. Geophys. Res., 111, C09024, doi:10.1029/2005JC003367, 2006.

Lundgren, P., Janson, S., Jonasson, S., Singer, A., and Bergman B.: Unveiling of Novel Radiations within Trichodesmium Cluster by hetR Gene Sequence Analysis, App. Environm. Microb., 71, 190-196, 2005.

Mahaffey, C., Michaels, A. F., and Capone, D. G.: The conundrum of marine $\mathrm{N}_{2}$ fixation, Amer. J. Sci., 305, 546-595, 2005.

Mantas, V. M., Pereira, A. J. S. C., and Morais, P. V.: Plumes of discolored water of volcanic origin and possible implications for algal communities. The case of the Home Reef eruption of 2006 (Tonga, Southwest Pacific Ocean), Remote Sens. Environ., 115, 1341-1352, 2011.

Maritorena, S. and Siegel, D. A.: Consistent merging of satellite ocean color data sets using a bio-optical model. Remote Sensing Environ., 94, 429-440, 2005.

Masotti, I., Ruiz-Pino, D., and Le Bouteiller, A.: Photosynthetic characteristics of Trichodesmium in the southwest Pacific Ocean: importance and significance, Mar. Ecol.-Prog. Ser., 338, 37-49, 2007.

McKinna, L. I. W., Furnas, M. J., and Ridd, P. V.: A simple, binary classification algorithm for the detection of Trichodesmium spp. within the Great Barrier Reef using MODIS imagery, Limnol. Oceanogr. Methods, 9, 50-66, doi:10.4319/lom.2010.9.50, 2011.

Moisander, P. H., Beinart, R. A., Hewson, I. White, A. E., Johnson, K. S., and Carlson, C. A.: Unicellular Cyanobacterial Distributions Broaden the Oceanic $\mathrm{N}_{2}$ Fixation Domain, Science, 327, 1512-1514, doi:10.1126/science.1185468, 2010.

Monteiro, F. M., Follows, M. J., and Dutkiewicz, S.: Distribution of diverse nitrogen fixers in the global ocean, Global Biogeochem. Cy., 24, GB3017, doi:10.1029/2009GB003731, 2010.

Montoya, J., Holl, C. M., Zehr, J. P., Hansen, A., Villareal, T. A., and Capone, D. G.: High Rates of $\mathrm{N}_{2}$ Fixation by Unicellular Diazotrophs in the Oligotrophic Pacific, Nature, 430, 1027-1032, 2004.

Moutin, T., Van den Broeck, N., Beker, B., Dupouy, C., Rimmelin, P., and Le Bouteiller, A.: Phosphate availability controls Trichodesmium spp. biomass in the SW Pacific Ocean, Mar. Ecol.Prog. Ser., 207, 15-21, 2005.

Mulholland, M. R.: The fate of nitrogen fixed by diazotrophs in the ocean, Biogeosciences, 4, 37-51, doi:10.5194/bg-4-37-2007, 2007.

Neveux, J., Lantoine, F., Vaulot, D., Marie, D., and Blanchot, J.: Phycoerythrins in the southern tropical and equatorial Pacific Ocean: Evidence for new cyanobacterial types, J. Geophys. Res., 104, 3311-3321, 1999. 
Neveux, J., Tenório, M. M. B., Dupouy, C., and Villareal, T.: Spectral diversity of phycoerythrins and diazotrophs abundance in tropical South Pacific, Limnol. Oceanogr., 51, 4, 1689-1698, 2006.

Qu, T. and Lindstrom, E. J.: A climatological interpretation of the circulation in the western south Pacific, J. Phys. Oceanogr., 32, 2492-2508, 2002.

Ramos, A. G. R., Martel, A., Codd, G. A., Soler, E., Coca, J., Redondo, A., Morrison, L. F., Metcalf, J. S., Ojeda, A., Duarez, S., and Petit, M.: Bloom of the marine diazotrophic cyanobacterium Trichodesmium erythraeum in the Northwest African Upwelling, Mar. Ecol.-Prog. Ser., 301, 303-305, 2005.

Revelante, N. and Gilmartin, M.: Dynamics of phytoplankton in the Great Barrier Reef lagoon, J. Plankton Res., 4, 47-76, 198, 1982.

Riemann, L., Farnelid, H., and Steward, G. F.: Nitrogenase genes in non-cyanobacterial plankton: prevalence, diversity and regulation in marine waters, Aquat. Microb. Ecol., 61, 235-247, doi:10.3354/ame01431, 2010.

Rubin, M., Berman-Frank, I., and Shaked, H.: Dust- and mineraliron utilization by the marine dinitrogen-fixer Trichodesmium. Nature Geosci., 4, 529-534, doi:10.1038/ngeo1181, 2011.

Sarangi, R. K., Chauhan, P., and Nayak, S. R.: Detection and monitoring of Trichodesmium blooms in the coastal waters off Saurashtra coast, India using IRS-P4 OCM data, Curr. Sci., 86, 12, 1636-1641, 2004.

Sato, M., Hashihama, F., Kitajima, S., Takeda, S., and Furuya, K.: Distribution of nano-sized cyanobacteria in the western and central Pacific Ocean, Aquatic Microb. Ecol., 59, 273-282, 2010.

Shiozaki, T., Furuya, K., Kodama, T., and Takeda, S.: Contribution of $\mathrm{N}_{2}$ fixation to new production in the western North Pacific Ocean along $155^{\circ}$ E, Mar. Ecol.-Prog. Ser., 377, 19-32, 2009.

Siegel, D. A., Maritorena, S., Nelson, N. B., Hansell, D. A., and Lorenzi-Kayser, M.: Global distribution and dynamics of colored dissolved and detrital organic materials, J. Geophys. Res., 107, 3228, doi:10.1029/2001JC000965, 2002.
Siegel, D. A., Maritorena, S., Nelson, N. B., and Behrenfeld, M. J.: Independence and interdependencies of global ocean color properties: Reassessing the bio-optical assumption, J. Geophys. Res., 110, C07011, doi:10.1029/2004JC002527, 2005.

Subramaniam, A., Carpenter, E. J., Karentz, D., and Falkowski, P. G.: Bio-optical properties of the diazotrophic cyanobacteria Trichodesmium spp. I. Absorption and photosynthetic action spectra, Limnol. Oceanogr., 44, 608-617, 1999.

Subramaniam, A., Brown, C. W., Hood, R. R., Carpenter, E. J., and Capone, D. G.: Detecting Trichodesmium blooms in SeaWiFS imagery, Deep-Sea Res. Pt. I, 49, 107-121, 2002.

Tadokoro, K., Ono, T., Yasuda, I., Osafune, S., Shiomoto, A., and Sugisaki, H.: Possible mechanisms of decadal scale variation in $\mathrm{PO}_{4}$ concentration in the western North Pacific, Geophys. Res. Lett., 36, L08606, doi:10.1029/2009GL037327, 2009.

Tenório, M. M. B. : Les cyanobactéries en milieu tropical: occurrence, distribution, écologie et dynamique, $\mathrm{PhD}$ Thesis, Université Paris VI, 2006.

Westberry, T. K. and Siegel, D. A.: Spatial and temporal distribution of Trichodesmium blooms in the world's oceans, Global Biogeochem. Cy., 20, 4016, doi:10.1029/2005GB002673, 2006.

Westberry, T., Subramaniam, A., and Siegel, D.: An improved bio-optical algorithm for the remote sensing of Trichodesmium spp. blooms, J. Geophys. Res., 110, C06012, doi:10.1029/2004JC, 2005.

Wilson, C. and Qiu, X.: Global distribution of summer chlorophyll blooms in the oligotrophic gyres, Prog. Oceanogr., 78, 107-134, doi:10.1016/j.pocean.2008.05.002, 2008.

Zehr, J. P. and Kudela, R. M.: Nitrogen cycle of the open ocean: from genes to ecosystems, Annu. Rev. Marine Sci., 3, 197-225, 2011.

Zehr, J. P., Waterbury, J. B., Turner, P. J., Montoya, J. P., Omoregie, E., Steward, G. F., Hansen, A., and Karl, D. M.: Unicellular cyanobacteria fix $\mathrm{N}_{2}$ in the subtropical North Pacific Ocean, Nature, 412, 635-638, doi:10.1038/35088063, 2001. 